\title{
An ERP study to investigate age-related differences in sensory gating and auditory processing.
}

\author{
by
}

Caidence Paleske

A thesis submitted to the Faculty of Graduate and Postdoctoral Affairs in partial fulfillment of the requirements for the degree of

Master of Arts

in

Psychology

Carleton University

Ottawa, Ontario

(C) 2019

Caidence Paleske 


\begin{abstract}
Sensory gating is a mechanism that inhibits neurological responses to redundant information. Although studied within clinical populations, sensory gating has rarely been investigated with respect to aging. The present research investigated age-related changes in auditory sensory gating using electroencephalography and quantified the effect of age on adaptive gating responses for oddball stimuli. Age-related differences in sensory gating and oddball responses were observed in late-stage auditory processing, where older adults exhibited weaker sensory gating. An attenuated P200 for first and second clicks seen in the aging group may explain the weaker sensory gating ratio seen in older participants. Furthermore, replacing the second click with oddball stimuli attenuated, but did not eliminate sensory gating, indicating that sensory gating is a dynamic mechanism. Findings suggest that sensory gating is diminished for older adults and may transform from an adaptive mechanism under executive control to a pre-attentive limiter of auditory processing.
\end{abstract}




\section{Acknowledgements}

This thesis is dedicated to those individuals with which this document would not have been possible. Firstly, I would like to thank and acknowledge my committee for their feedback and advisement. Secondly, to my supervisor, Chris Herdman, for creating a lab where the focus is not only on executing fantastic research, but on how research findings can make an everlasting impact on real-world issues. I am so grateful to have been a member of the Advanced Cognitive Engineering (ACE) Lab, and have continued my passion for applied cognitive neuropsychology with my current position at the National Research Council.

I am also forever in debt to Kathy Van Benthem, whose patience and guidance made this thesis possible. Thank you, Kathy, for your many hours spent helping me to connect the dots, and for taking the time to integrate me into the world of aviation research. I would also like to thank the National Research Council, for playing a role in this research, and for continuing to put incredible value in the physiological assessment of behavior within the aviation industry. And a heart-felt thank you to Heather WrightBeatty, for providing me the opportunity to continue to work towards the completion of my degree, despite starting a full-time job within the Human Factors group this past January.

Thank you to my Mum, Deb Roy, and sisters, Santana Paleske and Scarlett Schumacher, who have always been incredibly supportive of my journey and stood by my side through every step, despite the distance and crazy ideas. And finally, to Jesse Lock, who has stood by my side through my best and worst times. As we close the door 
on both of our Masters degrees, I wish that we continue to enjoy the music of life, and may we always find time to appreciate the push + pull of the journey. 


\section{Table of Contents}

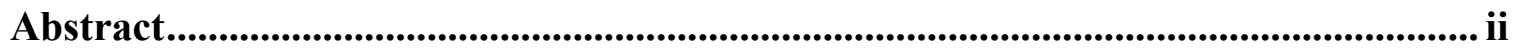

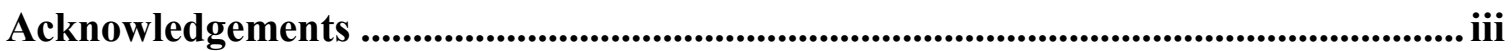

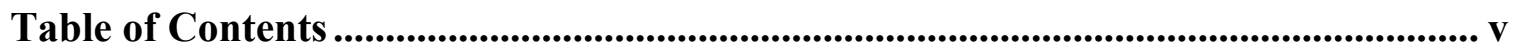

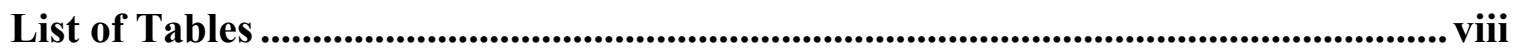

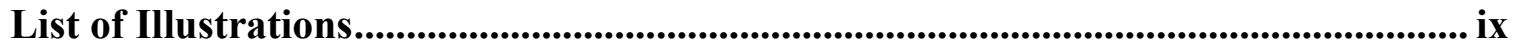

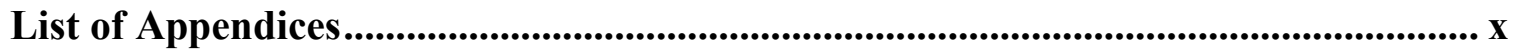

Chapter 1: Introduction ......................................................................................... 1

Chapter 2: Literature Review .......................................................................................... 4

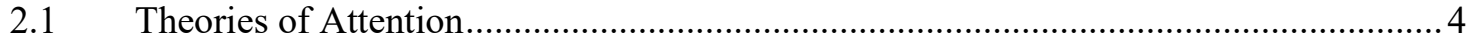

2.1.1 Pre-attentive stage of information filtering ..................................................... 4

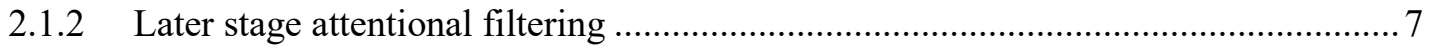

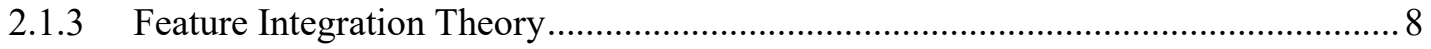

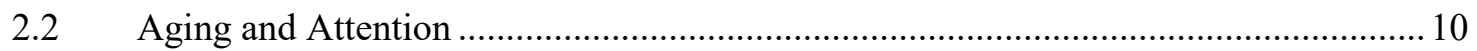





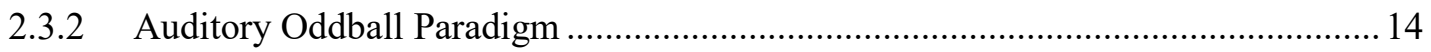

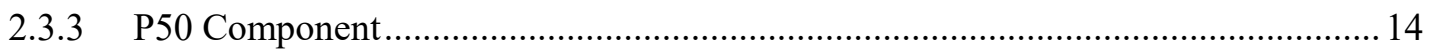

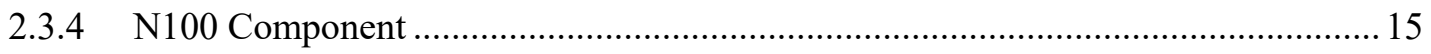

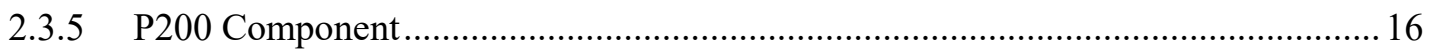

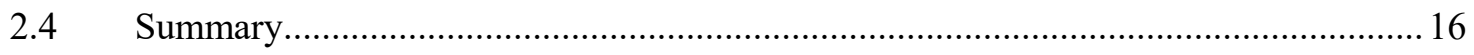

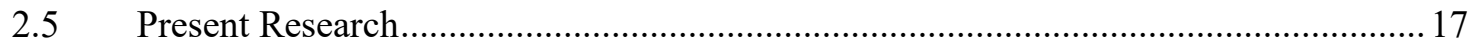

Chapter 3: Methods .................................................................................................................... 20

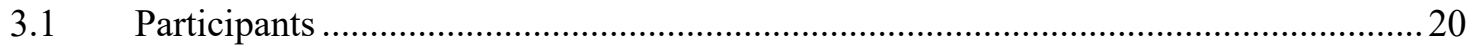

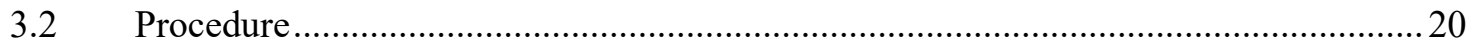




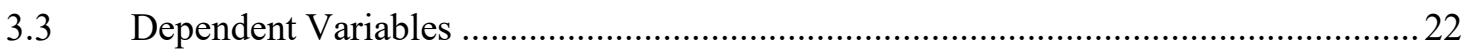

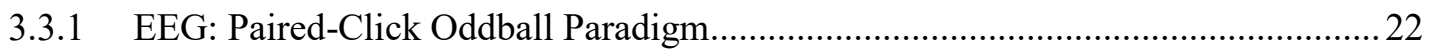

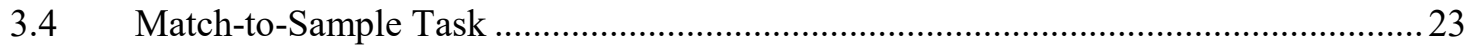

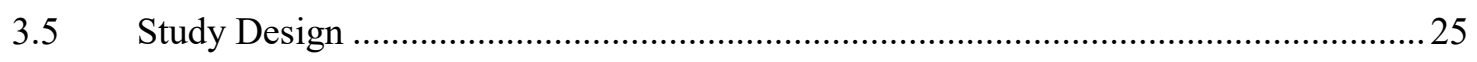

3.5.1 EEG Procedures and Evoked Potential Procedures .............................................25

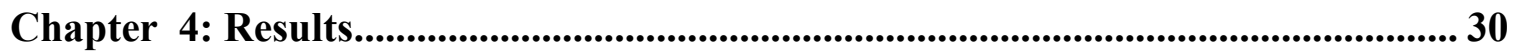

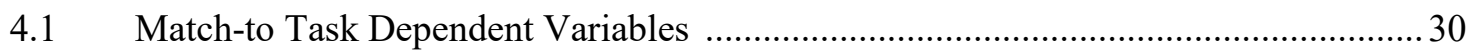

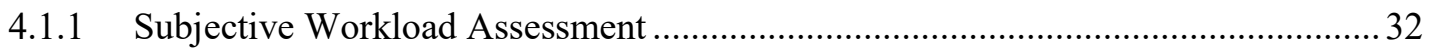

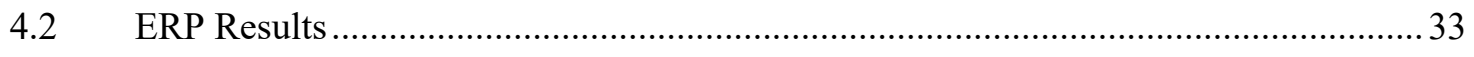



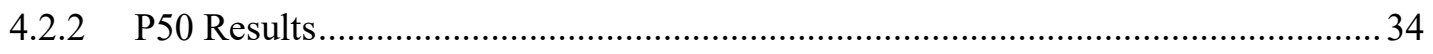

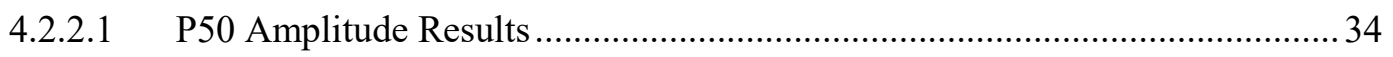

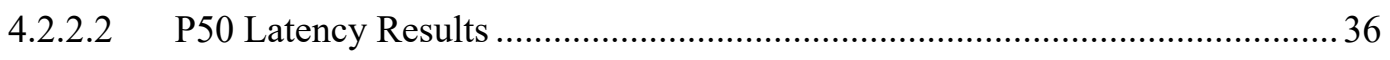

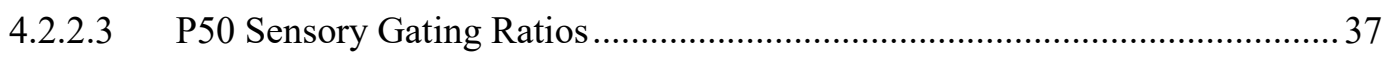

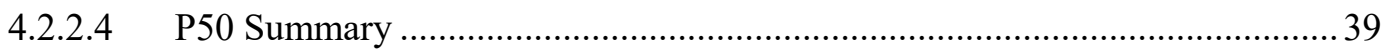

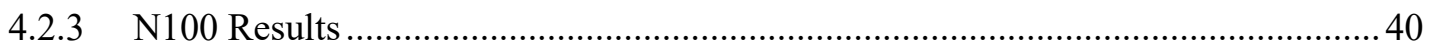

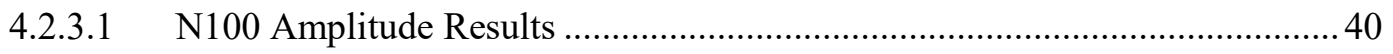

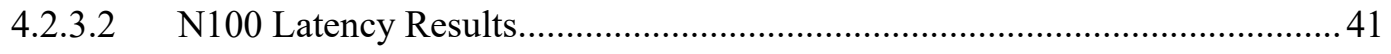

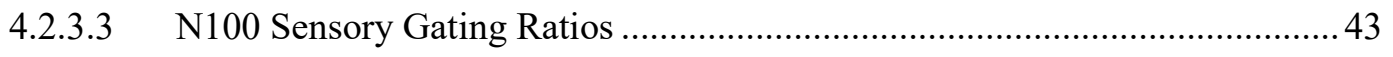



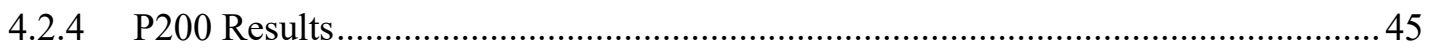

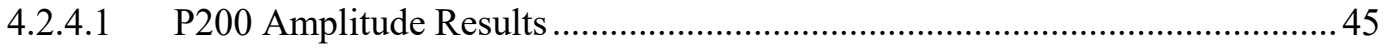

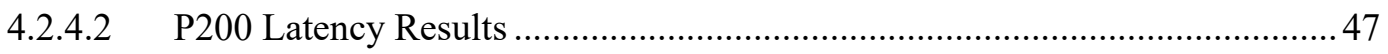

4.2.4.3 P200 Sensory Gating Ratios ................................................................... 49

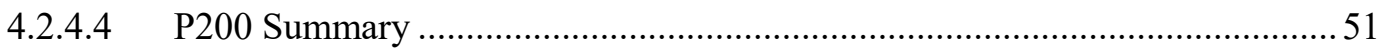

4.3 Correlations between Sensory Gating Ratios and Match-To Performance ..................52 


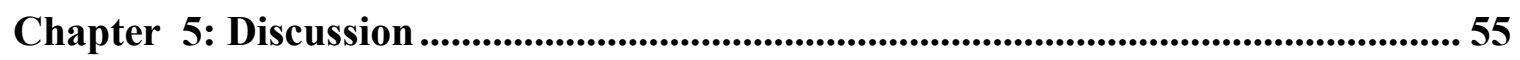

5.1 Age influenced sensory gating ratios during attentive stages of processing ….............55

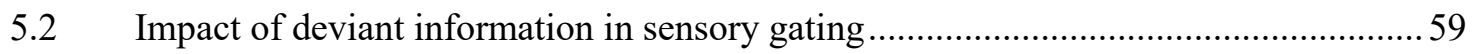

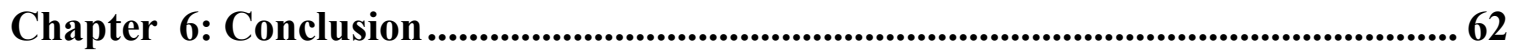

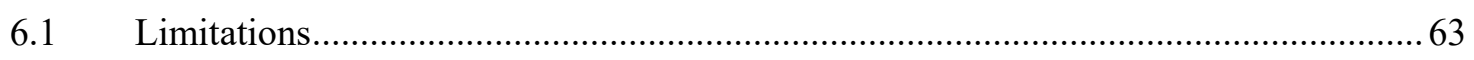

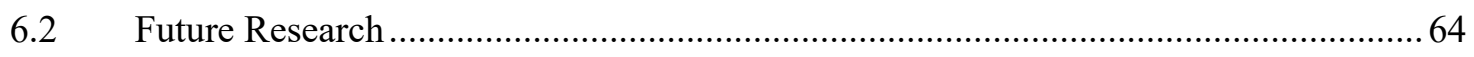

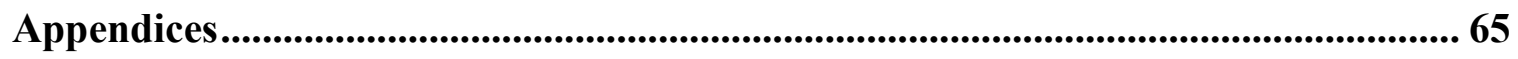

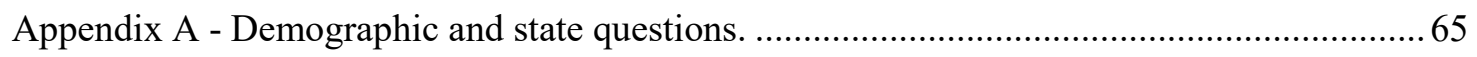

Ethics Clearance Form .........................................................................................66

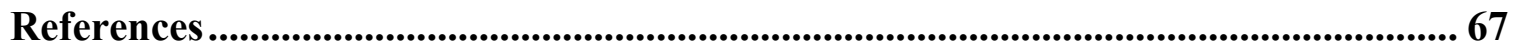




\section{List of Tables}

Table 1. Descriptive Results for the Number of Correctly Classified Grids ANOVA

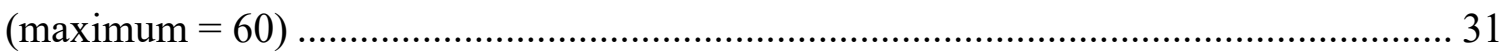

Table 2. Mean S1 and S2 amplitudes during baseline for each ERP, separated by age... 34

Table 3. Summarized significant effects for each ERP dependent variable...................... 54 


\section{List of Illustrations}

Figure 1. Neurological pathway for auditory information. Adapted from "The linguistic benefits of musical abilities", by Patel, A.D. \& Iversen, J.R., 2007, Trends in Cognitive

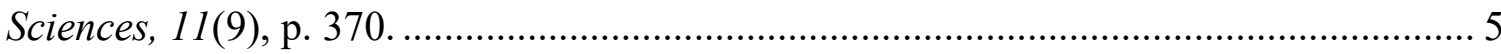

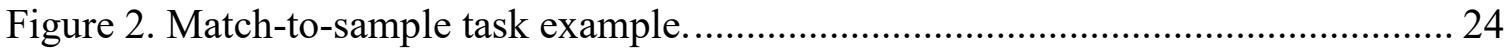

Figure 3. Averaged ERP response for a single participant with peaks and troughs identified with black vertical lines.............................................................................. 28

Figure 4. Mean reaction time for each age group, separated by workload condition.Easy

Figure 5. P50 amplitude by click type, separated by age group and workload. ............... 35

Figure 6. P50 latency by click type, separated by age group and workload..................... 37

Figure 7. Mean P50 gating ratio by gating condition and workload. .............................. 38

Figure 8. P50 gating ratio by workload, separated by age group and gating condition.... 39

Figure 9. N100 amplitude by click type, separated by age group and workload.............. 40

Figure 10. N100 latencies by click type and age group.................................................. 42

Figure 11. N100 latencies by click type, separated by age group and workload.............. 43

Figure 12. N100 gating ratio by workload, separated by age group and gating condition.

Figure 13. P200 amplitude by click type, separated by age group and workload. ........... 46

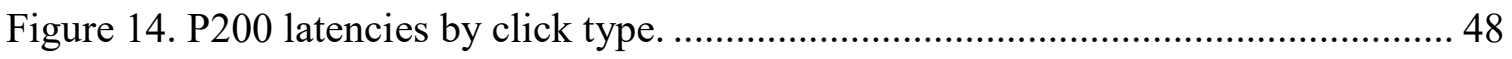

Figure 15. P200 latencies by click type, separated by age group and workload. ............. 49

Figure 16. P200 gating ratios by workload, separated by age group and gating condition. 


\section{List of Appendices}

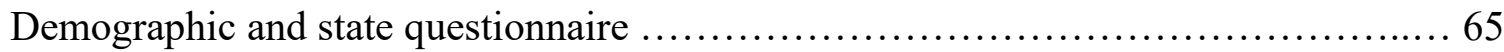




\section{Chapter 1: Introduction}

As we experience our daily life, our nervous system is continuously bombarded with a wide range of stimuli. Although sensory systems are able to encode and briefly retain vast amounts of information, the processing capacity of higher-level cognitive systems is limited. Accordingly, stimuli that enter the sensory system must be prioritized and selected for further processing. Selection of stimuli occurs along a continuum of neural processes, from a pre-attentive stage to a later stage involving attention. Sensory gating is an important early stage mechanism for ensuring that attentional resources are applied advantageously. Sensory gating is the inhibition of neurological responses to redundant information, and is thought to be advantageous because it protects higher order cognitive functions from being overloaded with irrelevant sensory stimuli (Kisley, Noecker, \& Guinther, 2004; Lijffijt et al., 2009). Sensory gating efficiency is known to be sensitive to individual differences, such as age (Boutros et al., 2000; Fuerst, Gallinat, \& Boutros, 2007) and mental health (Adler, et al., 1982; Heinrichs, 2004).

Electroencephalography (EEG) is the primary method that researchers have used to examine the neurological processes involved in sensory gating. EEG technology provides a temporal history of the phases involved in processing a given stimulus and supports the investigation of attention processes at the level of a millisecond. However, the majority of what we know about sensory gating has come from studying clinical populations, with minimal investigation on how sensory gating changes over the course of a lifetime.

In the presented research, I used EEG data collected using a novel paradigm to investigate the effects of age on early and middle stages of auditory processing, and 
quantified the effect of age on sensory gating for both relevant and irrelevant stimuli. In this work, auditory processing takes place against a background of low and high visualspatial working memory activities to permit the study of sensory gating within simulated real-world experiences.

A significant amount of research using a paired click paradigm has examined how schizophrenia impacts sensory gating, demonstrating that those diagnosed have weaker sensory gating compared to healthy controls (Adler et al., 1982; Franks, Adler, Waldo, Alpert, \& Freedman, 1983; Thomas et al., 2010; Lijffijt et al., 2009). However, less research has looked directly into the impact of aging on sensory gating. In one example, researchers investigated the hypothesis that older adults and those diagnosed with alcoholism would show similar sensory gating patterns (Boutros et al., 2000). Older adults were found to exhibit weaker sensory gating compared to healthy controls, but only for late-stage attentive processing (i.e., the P300 event-related potential). Another study that sought to investigate age-related differences in sensory gating found contradictory results, with no age differences during pre-attentive or attentive stage processing (Gmehlin, Kreisel, Bachmann, Weisbrod, \& Thomas, 2011). Given contradictory evidence within a small number of studies, further investigation was warranted.

The goal of the present research was to examine how normal aging influences auditory sensory gating, and how increasing the saliency of otherwise redundant stimuli modulates the sensory gating response for older and younger participants. To do this, a paired click and oddball design were combined into a paired click oddball paradigm (PCOP). Classic paired click paradigms involve presenting two identical clicks within 
short succession of each other. The brain interprets the second click as redundant information and suppresses the neurological response to that stimulus. Sensory gating is an automatic neurophysiological response, and does not have to be learned with repeated presentation. Suppression of redundant stimuli creates efficiency, and prevents informational overload. Because the work on aging and sensory gating is limited, hypotheses surrounding the outcomes of the PCOP are based primarily on how older age typically impacts event-related potentials (ERPs). It was expected that older adults would demonstrate reduced sensory gating when compared to younger adults for the conventional paired click task, in addition to weaker sensory gating for deviant stimuli. There is an exploratory element to this study, in that how sensory gating will be affected by both age and a deviant stimuli is a new research question. By examining age-related differences in sensory gating, this thesis will contribute to the sensory gating literature and will provide suggestions as to how age differences in auditory attention can be attenuated by incorporating salient sounds in a complex environment. 


\section{Chapter 2: Literature Review}

The following chapter discusses theories of attention, how auditory attention is typically researched, the mechanism of sensory gating, and the current state of the literature with respect to aging and auditory attention. The neurophysiological responses that are typically associated with early and late stages of attention are discussed, as well as the key cortical areas involved during attentional processes.

\subsection{Theories of Attention}

The central nervous system is constantly bombarded with a wide variety of stimuli that our brains must efficiently process. Given this problem, the brain has adapted neurological mechanisms for filtering out repetitive or unimportant stimuli, while also selecting relevant stimuli for further processing. Though the use of conscious and unconscious attentional mechanisms, the selection of stimuli for further processing depends, in part, on task goals. While immersed in a highly dynamic environment, the brain cannot only rely on conscious attentional mechanisms. Because the use of conscious mechanisms have been shown to miss important stimuli, it is important to understand how and why certain types of stimuli are allowed through our neurological filter. In this chapter, theories related to pre-attentive and attentive filtering are discussed and related to the research question of age-related changes in auditory sensory gating.

\subsubsection{Pre-attentive stage of information filtering}

Auditory information has a known neurological pathway, originating from the auditory nerve and extending to the auditory cortex within the temporal lobes (Figure 1). Upon reaching the auditory cortex, information is then sent through an interconnected network throughout the cortex for further processing. Processing streams for visual 
information have been extensively studied and are well defined; the ventral and dorsal processing streams within the cortex account for the "where" and "what" of visual information (Goodale \& Milner, 1992). Despite being separate modalities, auditory down-stream processes are known to excite similar cortical regions compared to those involved in down-stream processing in vision, such as the inferior parietal cortex, the superior temporal gyrus, and dorsolateral prefrontal cortex (Hackett, 2012; Rauschecker \& Tian, 2000).

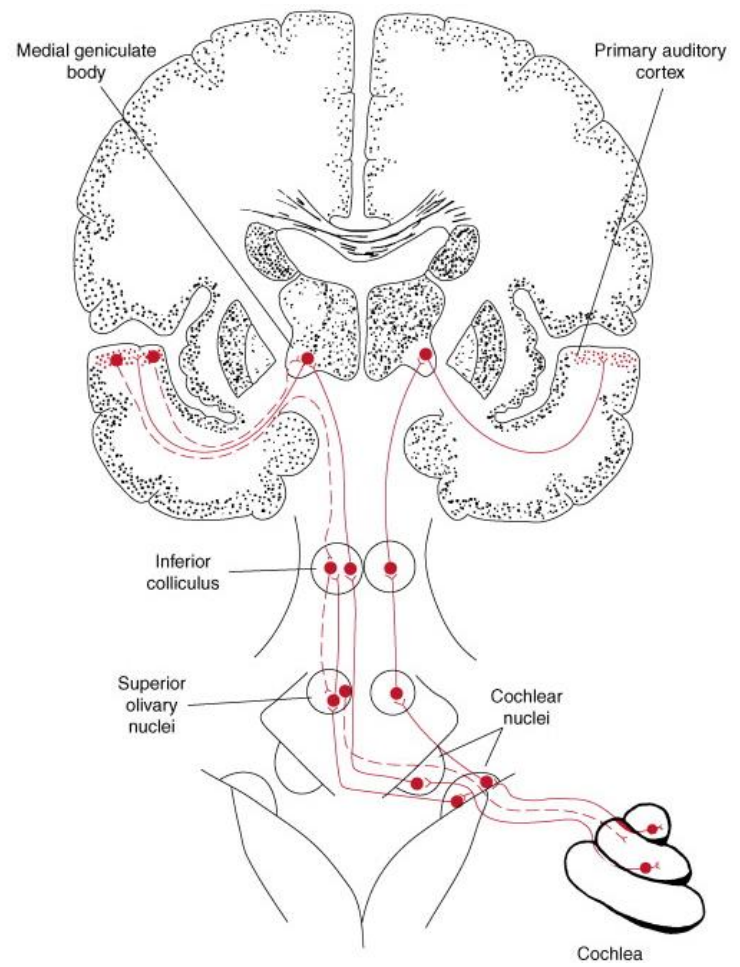

Figure 1. Neurological pathway for auditory information. Adapted from "The linguistic benefits of musical abilities", by Patel, A.D. \& Iversen, J.R., 2007, Trends in Cognitive Sciences, 11(9), p. 370. In 1958, Broadbent proposed a model of selective attention in which a neurological "filter" selects relevant information based on task demands and priorities. Information that successfully passes through the selective filter is made available for further processing. In sum, in Broadbent's model, the selective filter is an early gating 
mechanism that allows information that is deemed relevant to be selectively attended and made available to higher-level cognitive processes. Information that is not attended is not processed beyond sensory memory.

Broadbent (1958) was able to demonstrate this filter mechanism through a dichotic listening task in which participants were required to attend to one of two streams of auditory information that were presented simultaneously. Participants had better recall for the attended stream, and lower accuracy for the unattended stream. It was later demonstrated that visual information is only held in sensory memory for around 500 milliseconds (Erikson \& Collins, 1967). Although sensory memory systems have very large capacities (Sperling, 1960), only a small amount of information can be passed on to short-term memory, which has a limited capacity. The selection of information from sensory memory must happen very quickly, as information is only briefly held. According to Broadbent, this action of filtering information is based on the physical characteristics of the incoming stimuli, such as colour, location, and primitive features like pitch, lines, and curves. He therefore proposed that filtering mechanisms are applied during very early stages of auditory processing, before the full semantic meaning of the stimuli is interpreted (Broadbent, 1958).

Broadbent's interpretation of filtering was expanded in Treisman's (1960) model of attention. Treisman proposed that filtering acts not as an all-or-none mechanism, but that thresholds differ between the unattended and attended-to channel. She proposed that the unattended channel is merely attenuated, not blocked, from further processing, and that attenuation can be lessened if the incoming stimuli is deemed important. Using a similar dichotic listening task, Treisman demonstrated that "important" stimuli, such as 
one's name or maintaining sentence context, have the ability to automatically redirect our attention to the unattended ear because the threshold for these types of stimuli are lower. The ability of contextual cues to lower the threshold for attention goes against Broadbent's theory, which stated that unattended information is blocked from passing through the filter, and that filtering occurs prior to the assignment of importance (Treisman, 1960). Treisman went on to extend her work on attentional filtering to propose feature integration theory, discussed in Section 2.1.3.

\subsubsection{Later stage attentional filtering}

Late stage selection of stimuli occurs based on the further processing of the physical or semantic properties of the stimulus as a means for decision making (Alperin et al., 2013). One example of late stage attentional filtering is selective attention. Selective attention is a top-down modulation mechanism that facilitates the processing of task-relevant stimuli over and above task-irrelevant stimuli (Daffner et al., 2012). Because of prioritization, selective attention is thought to improve the processing of relevant stimuli while conserving cognitive resources for higher order decision making (Daffner et al., 2012; Gazzaley, Cooney, McEvoy, Knight, \& D'Esposito, 2005).

Studying selective attention within the field of cognitive neuropsychology typically involves the comparison of neural activity during attended stimuli versus unattended stimuli. Auditory selective attention studies have demonstrated that the amplitudes for N100 and P200 ERPs tend to increase for attended-to compared to ignored auditory stimuli, but that this increase is less pronounced in older adults (Karayanidis, Andrews, Ward, \& Michie, 1995). Whether the source of age-related declines in selective attention 
is occurring during pre-attentive or attentive stages of auditory processing is a question currently being asked by researchers, and the presented work.

\subsubsection{Feature Integration Theory}

Our everyday environment is complex and requires that we continuously shift our attention from one modality to another. However, we are limited in our capacity to store accessible information. This constraint proposes a challenge, where features from separate modalities must be first combined within their own modality, and then integrated into a holistic interpretation of the environment. Feature processing, as described by Treisman and Gelade (1980), occurs in two stages: pre-attentive and attentive. Although the pre-attentive stage occurs prior to conscious awareness, feature processing during this stage can guide the subsequent direction of attention (Treisman \& Gelade, 1980). Both auditory and visual objects contain a variety of base-level features, including pitch and timbre, or color, line direction, and contrast. Research has provided evidence that auditory features such as pitch and timbre are processed pre-attentively, similar to basic properties of visual stimuli, and are then combined into a percept that is acknowledged via attention (Hall, Pastore, Acker, Huang, 2000). Auditory processing is notoriously difficult to assess using behavioural outcomes, which only provide an overt description of the mechanisms involved in a theory that is dependent on neurocognitive processes. In order to better understand the neural correlates underlying auditory feature integration, biophysiological technologies such as electroencephalography (EEG) and functional magnetic resonance imaging (fMRI) can be used.

Research using EEG has identified neural responses associated with both preattentive and attentive responses to auditory stimuli. Neural responses to a specific 
stimulus are known as event-related potentials (ERPs), and are measured as small positive or negative voltage changes generated within the brain (Sinha \& Sur, 2009). Processing auditory stimuli has distinct ERPs, such as the P50, N100, and the P200, that occur in response to the presentation of a discrete external stimulus. Characteristics of ERPs, such as amplitude and latency, can be modulated by mental health diagnoses (Boutros et al., 2012), workload (Van Benthem, Cebulski, Herdman, \& Keillor, 2017), and age (Gmehlin et al., 2011).

The P50 is a small positive deflection occurring approximately 50 milliseconds following stimulus presentation, and is regarded as a pre-attentive marker for auditory processing. The pre-attentive P50 is then followed by what are regarded as attentive ERPs (e.g., N100 and P200). The N100 and P200 can be influenced by attention, and are typically enhanced when attention is directed at target stimuli (Karayanidis et al., 1995). The sequential occurrence of pre-attentive and attentive ERPs in response to an auditory stimulus is similar to the two-stage process described within feature integration theory. However, multi-sensory environments pose additional challenges for feature integration: what are the criteria that permit certain stimuli to be successfully integrated, and others to be filtered? Coping with complex environments involves cross-modal competition and the ability to direct attention to task-relevant stimuli (Grant \& Weissman, 2017). However, an increase in environmental complexity does not mean that task-irrelevant stimuli are not processed. This follows Treisman's model of attention, which suggests that task-irrelevant stimuli are still processed, just not to the same degree as task-relevant stimuli (Treisman, 1960). Selective attention processes within complex environments 
permit an attentive bias towards processing task-relevant stimuli, which has been demonstrated to activate key areas within the cortex.

Previous studies have highlighted the importance of the frontal and parietal cortices as part of a network that is involved in coordinating attention during dual-modal tasks (Moisala, et al., 2015). Activity within the inferior frontal cortex (Herath, Klingberg, Young, Amunts, \& Roland, 2001), the dorsolateral and medial prefrontal cortex (D'Esposito, Detre, Alsop, Shin, Atlas, \& Grossman, 1995), the superior parietal lobule (Yoo, Paralkar, \& Panych, 2004), and the right temporoparietal junction (Corbetta \& Shulman, 2002) have been shown to correlate with performance during selective and divided attention tasks. Greater blood-oxygen-level dependent responses within these regions has been proposed to indicate competition for resources during cross-modal tasks (Moisala et al., 2015), and that cross-modal interference between sensory cortices is not solely responsible for decreases in performance during high workload cross-modal scenarios.

\subsection{Aging and Attention}

Research has demonstrated age-related declines in executive functioning, including attention switching, inhibition, and updating (McAlister \& SchmitterEdgecombe, 2016). One possible explanation for these observations is the frontal aging hypothesis, which states that the frontal lobes degenerate with the normal aging process (Dempster, 1992; Rodríguez-Aranda \& Sundet, 2006). Despite a reduction in frontal lobe activity, older adults, to their own detriment, tend to rely more on the frontal lobes rather than parietal cortices to complete complex tasks (Davis et al., 2008; Grady et al., 1994; O’Connell et al., 2012). Older adults may also tend to recruit more frontal areas rather 
than the traditional temporal-parietal areas when processing deviant information, despite experiencing a degradation of the frontal lobes (Fjell \& Walhovd, 2001; Li, Gratton, Fabiani, \& Knight, 2013). This migration of neural recruitment is known as the posterioranterior shift in aging, abbreviated PASA. Other similar theories such as the hemispheric asymmetry reduction in older adults (HAROLD) (Cabeza, 2002) and the compensationrelated utilization of neural circuits hypothesis (CRUNCH) (Reuter-Lorenz \& Cappell, 2008) also attempt to explain the neurological underpinnings of age-related deficits in executive functioning.

Although numerous studies have found support for decreased executive functioning in older adults, the evidence for an age-related effect in cross-modal tasks is less clear. One domain of research demonstrates that age-related reductions in crossmodal task performance occur only when the target stimulus is auditory and the distractor is visual (Guerreiro \& Van Gerven, 2011), which suggests that age-related performance on cross-modal tasks depends on the modality of the target and distractor (Guerreio \& Van Gerven, 2015; Leiva, Parmentier, \& Andrés, 2015). Research has also demonstrated that although age has no impact on performance when the tasks are in same modality (i.e., a visual task with a visual distractor, or an auditory task with an auditory distractor) (Leiva \& Parmentier, 2015), selective attention declines with age during cross-modal tasks (Guerreiro, Anguera, Mishra, Van Gerven, \& Gazzaley, 2014; Puschmann, Sandmann, Bendixen, \& Thiel, 2014). Taken together, these research findings demonstrate a clear age-related detriment in allocation of attention in complex environments. But what this body of research does not demonstrate is whether or not management of cross-modal attention is a result of unattended information being pre- 
attentively filtered to manage the bottleneck phenomenon, or whether neurological responses to task-irrelevant stimuli are suppressed at the attentive level.

\subsection{Neurophysiology of Auditory Attention}

Attention is often studied using electroencephalography (EEG), either by examining how different task environments impact continuous brain waves, or by using discrete auditory or visual stimuli. EEG allows researchers to infer spatial and temporal patterns of cortical activity by recording minute electrical changes on the scalp. Neural processes that support auditory processing are frequently studied using sensory gating and oddball paradigms, and typically pre-attentive (P50 and N100) and attentive (P200400) components. It is important to remember that an individual ERP is the result of an accumulation of widespread neurological activity, and not only detected at a single point. Multiple cortical areas are involved in stimulus processing, and it is when their activities are summed and averaged across many trials that we observe the wave-like ERP associated with stimulus presentation.

\subsubsection{The Mechanism of Sensory Gating}

Sensory gating (SG) is a filtering mechanism whereby the processing of irrelevant or redundant information is attenuated by the central nervous system (Boutros et al., 2000). Sensory gating has been shown to occur at pre-attentive and later auditory processing stages, before comprehension. Sensory gating is frequently indexed as the ratio of neurological responses to two paired auditory clicks, S1 and S2. In other words, sensory gating ratios are calculated by taking the amplitude of the neurophysiological response of the second click over the first. Attenuated S2 responses result in smaller gating ratios, and therefore reflect better gating efficiency. The paired-click paradigm is a 
paradigm, typically passive, that involves presenting participants with two subsequent auditory clicks, with the second click conceptualized as irrelevant information due to redundancy. The first click captures attention, and the second click, due to the short interstimulus interval, results in an attenuated response in comparison to the first click. By suppressing neurological responses to the second click, the brain effectively filters out irrelevant information. Sensory gating is primarily investigated using EEG, with the primary electrode site in question being $\mathrm{Cz}$ (i.e., the vertex of the skull). One study utilizing fMRI demonstrated that key areas involved within the mechanism of sensory gating are the auditory and prefrontal cortices, but further work is needed that utilizes fMRI to study sensory gating (Mayer, et al., 2009).

Research has shown reliable evidence for a reduction in P50 sensory gating in schizophrenic patients (Adler et al., 1982; Franks et al., 1983), but the literature is not well established for older adults (Boutros, Zouridakis, \& Overall, 1991). Evidence for sensory gating across ERPs in older adults is mixed, with some evidence indicating weaker sensory gating for older adults during attentive but not pre-attentive P50 ERP components (Boutros, et al., 2000; Gmehlin et al., 2011). Although the paired-click paradigm taps into the capability of the brain to suppress extraneous information, oddball paradigms provide a methodology for investigating attentional processing of unexpected information. To further investigate the extent to which older adults differ from younger adults in early and mid-stage auditory processing, paired-click and oddball tasks were combined to create a novel method for simultaneously investigating sensory gating and auditory attention. 


\subsubsection{Auditory Oddball Paradigm}

Local changes in neural activation propagate through the brain as event-related potentials (ERPs), or small changes in voltage in response to a specific event or stimuli (Sur \& Sinha, 2009). Obligatory responses to frequent auditory stimuli include the P50, N100, and P200 components, which encapsulate the early, and mid to late stages of auditory processing. However, if infrequent stimuli (e.g., a higher pitched stimulus) are placed amongst frequently presented stimuli, the amplitude of the P200 can increase, as well as initiate another ERP known as the P300 (Crowley \& Colrain, 2004; Yurgil \& Golob, 2013). The aspect of oddball, or "deviant", is created based on the frequency of the stimuli presented when amongst other high probability stimuli. The enhanced neurological response is not due to conscious assignment of "deviant", but is an automatic response to a low probability stimulus.

Oddball and auditory click paradigms have been previously used to assess changes in mental workload, with longer latencies and decreased amplitudes for the N100 and P200/P300 ERPs during high workload tasks (Miller, Rietschel, McDonald, \& Hatfield, 2011; Solis-Marcos \& Kircher, 2019). However, amplitude and latency changes do not always scale linearly across ERPs, with some ERPs being more or less affected during low compared to high workloads (Solis-Marcos \& Kircher, 2019).

\subsubsection{P50 Component}

The P50 is a pre-attentive neurological response to an auditory stimulus. It is identified as a small positive deflection occurring around 50 milliseconds after stimulus presentation, but could occur between 35 and 80 milliseconds (Olincy \& Martin, 2005; Sur \& Sinha, 2009). A significant portion of research on P50 has been performed using 
sensory gating methodologies in clinical populations, demonstrating that individuals diagnosed with schizophrenia (Adler et al., 1982; Franks, et al., 1983), Alzheimer dementia (Thomas, et al., 2010), and personality and bipolar disorders (Franks, et al., 1983; Lijffijt et al., 2009) have a reduced sensory gating mechanism at the level of the P50. In fact, the relationship between insufficient sensory gating and schizophrenia is so consistent within the literature, that it is a trusted neurological marker of the diagnosis (Bramon, Rabe-Hesketh, Sham, Murray, \& Frangou, 2004; Heinrichs, 2004). However, the literature on P50 sensory gating and aging is sparse and has provided mixed results. Further, the vast majority of sensory gating studies involve participants simply sitting and listening to the auditory stimuli. Although this practice can demonstrate how sensory gating operates as a baseline, it does not consider how sensory gating may be modified during a more realistic environment.

\subsubsection{N100 Component}

The N100 ERP is a neurological marker for auditory processing (Callan, et al., 2018; Hall, 1992). The N100 is a negative deflection that typically occurs between 80150 milliseconds following the onset of an unexpected auditory stimulus (Sur \& Sinha, 2009). Unlike P50, N100 is typically regarded as an ERP that can be influenced by attention (Grunwald et al., 2009). Research areas that investigate the modulation of the N100 include language processing (Weber-Fox, Leonard, Wray, \& Tomblin, 2010), selective attention (Hamilton, Baskin-Sommers, \& Newman, 2014), and classification of cognitive workload for brain-computer interfaces (Van Benthem et al., 2017). Although the sensory gating literature primarily investigates P50 because of its pre-attentive qualities, research has also demonstrated sensory gating at the level of the N100 
(Guterman, Josiassen, \& Bashore, 1992; Smith, Boutros, \& Schwarzkopf, 1994). It has been proposed that N100 gating could relate to filtering mechanisms associated with the triggering of attention (Boutros, Korzyukov, Jansen, Feingold, \& Bell, 2003; Lijffijt et al., 2009).

\subsubsection{P200 Component}

The P200 is a positive ERP deflection that occurs around $200 \mathrm{~ms}$, and has been shown to be influenced by selective attention, working memory, and aging (Buodo, Mento, Sarlo, \& Palomba, 2015; Lijffijt, et al., 2009; Lubitz, A.F., Niedeggen, M. \& Feser, M, 2017). Research has demonstrated that attention towards infrequent stimuli can decrease the amplitude of the P200 (Crowley \& Colrain, 2013), but how the gating response impacts the P200 when it is initiated by infrequent stimuli is a new area of research. Older adults tend to have attenuated P200 responses to auditory stimuli compared to young adults, and P200 amplitude can also be affected by increased workload (Daffner, et al., 2010; Lubitz et al., 2017). It is accepted that P200 amplitudes are associated with executive functioning processes, but it is less well understood how age impacts P200 amplitude, with some research pointing to a decrease in amplitude with age (Lubitz, et al. 2017, Daffner, et al., 2010) and others finding that an increase in amplitude with advancing age (Crowley \& Colrain, 2013; McEvoy, Pellouchoud, Smith, \& Gevins, 2001).

\subsection{Summary}

Researchers have demonstrated an age-related decline in attention related mechanisms. Theoretical rational and behavioural evidence have provided possibilities for how attention is modulated in the brain, during both pre-attentive and attentive stages 
of processing. Nearly five decades of research suggest that attention is a limited capacity mechanism, but the underlying neurological mechanisms for how and when filtering of stimuli occurs are still current research questions. Neurophysiological evidence using EEG corroborates the behavioural findings of selective attentional deficits, and supports the conclusion that these deficits are more pronounced in aging adults. Sensory gating is a possible neural mechanism that can help us further understand why aging adults may experience difficulty in the integration of pre-attentive and attentive information during multi-modal environments. Whether an age-related decline in selective attention to auditory stimuli is occurring as a result of pre-attentive or attentive neurological processing can be investigated with sensory gating. Further, in the presented research I modified the typical paired click design to include infrequently presented oddball stimuli as the second click in order to investigate whether the well-established mechanism of sensory gating is adaptable to stimulus properties.

\subsection{Present Research}

The present research investigated how the natural aging process impacts sensory gating, and how the gating mechanism adapts to deviant stimuli and to changes in workload. In order to investigate how sensory gating adapts to changes in stimulus presentation, the classic paired click paradigm was modified to include deviant stimuli, creating the paired-click oddball paradigm (PCOP). Sensitivity to changes in workload 
was investigated by manipulating task difficulty using a visual match-to task while participants listened to the passive PCOP task. Hypotheses are as follows:

1. Does aging influence sensory gating at pre-attentive or attentive stages of auditory processing?

a. Evidence for weaker gating in early, pre-attentive responses (i.e., P50) for older adults would indicate that more redundant information is being processed during early stages of auditory processing, and could be taking the place of what would otherwise be relevant stimuli within the very limited storage capacity system.

b. If older adults exhibit weaker gating during mid to late-stage ERP responses (i.e., N100 or P200), this could suggest that age-differences in sensory gating are more related to allocation of attention rather than preattentive perception.

2. Can the sensory gating mechanism be overridden by deviant stimuli?

a. If weaker sensory gating is seen within the deviant gating condition compared to the standard condition, then this would indicate that sensory gating is an adaptive mechanism that can be overridden by attentively relevant stimuli.

b. If older adults show weaker gating for deviant stimuli compared to younger adults, this would indicate that orienting attention is more easily 
triggered for older rather than younger adults when a stimulus is unexpected.

3. Sensory gating will be the weakest during hard workload.

a. Weaker sensory gating during hard workload would suggest that attention, either pre-attentively or attentively, is being focused on ensuring good performance on the visual task, which leaves less cognitive resources for actively suppressing auditory stimuli.

If sensory gating occurs during attentive but not pre-attentive processing, or vice versa, this could indicate that sensory gating serves different purposes for different stages of auditory processing. If sensory gating acts as a filtering mechanism during attentive stages of processing, then N100 and P200 sensory gating ratios could be influenced by workload, providing evidence that auditory sensory gating is adaptive. 


\section{Chapter 3: Methods}

\subsection{Participants}

A total of 30 participants (14 female) were recruited for this study, with 15 participants recruited through the university's online participation pool of undergraduate students (age: $\mathrm{M}=18.73$, range $=17-21$ ) to create the young adult group, and 15 participants above the age of 50 (age: $\mathrm{M}=67.27$, range $=52-75)$ recruited through campus posters and lab contacts. The age cut-off of 50 years old was decided based on previous research that demonstrated that pilots above the age of 50 years old had greater difficulty remembering auditory information, and had attenuated ERP component amplitudes compared to younger pilots. Both the young adult and older adult participants self-reported normal or corrected-to-normal hearing and vision. Inclusion criteria for the older adult group had the additional requirement of being at least 50 years old. Only right-handed participants were included for both age groups. Undergraduate participants received a $2 \%$ course credit, and the older adults were recruited as volunteers and provided with complimentary refreshments.

Sample size was calculated using the software GPower, and was based on the results of a meta-analysis on sensory gating (Rosburg, 2018), suggesting that a Cohen's effect size around 0.60 is adequate for detecting the effect of sensory gating. For the mixed factors design used in this experiment, a sample size of 30 was suggested in order to obtain a power estimate of around 0.8 .

\subsection{Procedure}

Participants were seated in front of a computer monitor while wearing a EEG headcap, with the response pad placed on the table in front of them. The experiment, 
including EEG set-up, practice trials and breaks, lasted approximately two hours. After completing of the experiment, the participant was debriefed and given $2 \%$ course credit (if recruited through SONA) or refreshments if a volunteer participant.

Prior to the experimental task, participants first answered a series of demographic and state related questions regarding alertness and hours of sleep (Appendix A), which was then followed by a 10-minute baseline EEG recording. During the baseline session, the PCOP paradigm was presented through a set of headphones and the participants were told to look at the black computer screen. Participants were told that they did not have to pay attention to the clicks throughout the experimental session. After completion of the baseline, participants received spoken instructions for how to complete the visual matchto-sample task.

Participants completed four blocks of the match-to-sample task, where easy and hard conditions were alternated (two blocks of each difficulty condition). Starting with either easy or hard blocks was counterbalanced between participants. Each block contained 30 trials of the match-to-sample task, and took between four and five minutes to complete. After the completion of each block, participants rated the difficulty of the block they just completed on a 5-point Likert scale ( 1 = Very Easy, 2 = Easy, $3=$ Neutral, $4=$ Hard, 5 = Very Hard). At the end of the fourth and final block, participants were asked if they used any specific strategies to help them succeed at the match-to task, and then they were debriefed. 


\subsection{Dependent Variables}

Within the match-to-sample task, response time for each trial and the number of correctly classified grids within each block was recorded. Subjective assessment of workload was assessed using the 5-point Likert scale described in Section 2.2.

\subsubsection{EEG: Paired-Click Oddball Paradigm}

Although participants performed a match-to-sample task on the computer, the key outcome of interest was the ERP responses within the paired-click oddball paradigm (PCOP). The PCOP is similar to the well-established paired click task, but with an additional oddball component. Two short clicks ( $3 \mathrm{~ms}$ each) separated by $500 \mathrm{~ms}$ were randomly presented every 2-4 seconds. An interpair interval (IPI) of 2-4 seconds was chosen based on the results of a meta-analysis, suggesting that the IPI for healthy adults in the paired click paradigm can be as short as 2 seconds (Dalecki et al., 2011). Paired clicks were presented to participants through a pair of headphones while they completed both the baseline and match-to-sample task.

The study design also included an auditory oddball paradigm in conjunction with the paired-click paradigm. By modifying the paired-click paradigm, an investigation as to how altering the pitch feature of the normally ignored second click moderated the expected paired click effect. By modifying the classic paired click task, it was possible to determine whether a deviant second click would eliminate (or lessen) the expected attenuation of the second click, and whether this recapturing of auditory attention by the use of a deviant second click was also evident in the older adults. To create this paradigm, oddball pairs were presented $15 \%$ of the time, and standard second clicks (i.e., the same pitch for both clicks) were presented $85 \%$ of the time. For the standard 
presentation, both clicks were presented at $1000 \mathrm{~Hz}$. For the deviant condition, the frequency of the first click was $1000 \mathrm{~Hz}$, whereas the frequency of the second click was $1500 \mathrm{~Hz}$. Both conditions are consistent in terms of interstimulus interval at 500 millisecond separation from the end of the first click to the start of the second click. The presentation of standard and deviant paired clicks was pseudo-randomized, such that from approximately 800 pairs of clicks throughout the course of the experimental task, $15 \%$ of all click pairs contained the oddball stimulus, the first three pairs of clicks from each group of 100 pairs were always standard clicks, and an oddball pair was always followed by two or more standard pairs. There was slight variation in the number of clicks each participant were exposed to because their time on-task is limited by the amount of time it took to complete each match-to-sample block.

\subsection{Match-to-Sample Task}

Participants performed a computerized match-to-sample task where they were required to match test and sample matrix patterns (Figure 2). In each trial, the participant saw a screen containing a single black and white matrix grid with a proportion of the cells shaded in, creating a matrix pattern. This study matrix was presented for 2000 milliseconds, which was then followed by a 2500 ms mask screen to prevent an after image. After the mask screen, a second matrix grid was displayed that either did or did not match the test grid. Participants were instructed to press the green button on the response pad if the second matrix was exactly the same as the first matrix, or to press a red button if the matrices differed. Response time was recorded. If the decision was not made within $3000 \mathrm{~ms}$, the word "Timeout" appeared on the screen, and that trial was 




Figure 2. Match-to-sample task example.

counted as an error. Task difficulty was manipulated by varying the number of shaded cells in each grid as well as the grid size. In the easy condition, a 5x5 matrix grid was presented with two of the 25 squares shaded black, while the remaining were white. In the hard condition, a 7x7 matrix grid was presented with seven of the 49 squares shaded black. Participants completed four blocks of the grid task with 30 trials in each. Block order was counterbalanced as either "easy, hard, easy, hard" or "hard, easy, hard, easy". After participants complete each block, they were asked to rate the difficulty of that block on a scale from 1 (very easy) to 5 (very hard). 


\subsection{Study Design}

A 3 (Workload: no task/baseline, low, high) by 3 (Click type: standard first S1, standard second S2, deviant second S2D) by 2 (Age: younger: $17-21$, older: $50-75$ ) mixed deisgn was used with repeated measures on the first two factors. Presentation of the easy and hard workload conditions was counterbalanced between participants in order to avoid any potential fatigue effects and subjective rating bias. Counterbalancing also helps to equalize changes in signal to noise ratios across the conditions over time, as this ratio is known to decrease over time with EEG measurements. Two reasons for this reduction are the gradual drying of wet electrodes, and electrode slippage against the skin, both of which result in unwanted noise within the data.

\subsubsection{EEG Procedures and Evoked Potential Procedures}

Each participant's head was measured in order to place a marker at the vertex of the skull for the $\mathrm{Cz}$ electrode. Vertex placement is calculated based on the cross-section created when taking the nasion to inion measure and the measurement from one preauricular process to the other. Prior to application, the headset was soaked in a saline solution made from potassium chloride, water, and mild (baby) shampoo as per the manual (Electrical Geodesics). Raw electroencephalogram (EEG) was recorded via a 128-channel EEG headcap (Electrical Geodesics Inc., Eugene OR, USA; EGI) with electrophysiological recordings being made via an Electrical Geodesics 200 series amplifier and electrode impedance below $60 \mathrm{~Hz}$. Electrodes were arranged in the international 10-20 system. EEG data was processed using the Matlab toolbox EEGLAB (Swartz Center for Computational Neuroscience, 2018). 
Signals were filtered off-line between 1 and $40 \mathrm{~Hz}$ to account for electrical noise, and channels were rejected based on a three standard deviation rule. Data was epoched using a $100 \mathrm{~ms}$ baseline prior to stimulus presentation and a 450 millisecond long epoch duration. Following epoch extraction, average re-referencing was computed. Independent components analysis was conducted using runica, followed by the multiple artifact rejection algorithm (MARA) for automated component rejection. MARA was chosen over other automated rejection machine learning algorithms because it has been trained on both manual classifications and well as machine classifications, (Winkler et al., 2011). The raw EEG file processing script used for this work was adapted from the methodology used in the Harvard Automated Processing Pipeline for EEG (HAPPE) algorithm, which is known for its ability to successfully reject component artifacts (e.g., muscle potentials, eye blinks) despite short recording sessions and a high number of recording channels (Gabard-Durnam, Mendez-Leal, Wilkinson, \& Levin, 2018).

Following component rejection, each participant's data was manually inspected. Darbeliai, an EEGLAB plugin, was used to visually inspect each file and identify each ERP. Two participants, one in each of the age groups, were removed from analysis due to abnormal activity seen after data processing was complete. Following inspection, ERP data was filtered at $10 \mathrm{~Hz}$ to examine the P50 and at $20 \mathrm{~Hz}$ for the N100 and P200 (Jerger, Biggins, \& Fein, 1992). This methodology has been shown to optimize detection of each separate ERP, especially for the P50 which is difficult to assess due to its small amplitude (Jerger et al., 1992; Lijffijt et al., 2009). ERP data was analyzed using peak-totrough comparisons as well as sensory gating ratios. Figure 3 shows an averaged ERP response for a single participant, with a $10 \mathrm{~Hz}$ high-pass filter applied in order to better 
detect the P50. The four black vertical lines identify the four peaks and troughs used to measure the amplitudes for each of the three ERPs. For the P50, amplitude was measured from the most negative trough preceding a positive deflection occurring between 30 and 80 milliseconds. The second vertical marker identifies the P50, with the first marker being the preceding trough. If the P50 was not present, then the amplitude for the N100 was measured as the amplitude from zero microvolts. The subsequent two markers after the P50 are the N100 and P200, with their amplitude measurements always being measured from the preceding marker (i.e., the N100 amplitude is measured with the preceding peak approximately as the P50 deflection). The N100 was always measured using the P50 as the preceding peak to the most negative trough occurring between 85 and 200 milliseconds. The P200 was measured from the N100 as a preceding trough to the most positive peak occurring between 150 and 250 milliseconds. Because separate high-pass filters were applied to the P50 and N100/P200, the preceding peak for the N100 is not the same amplitude as the P50 when the $10 \mathrm{~Hz}$ filter is applied. 


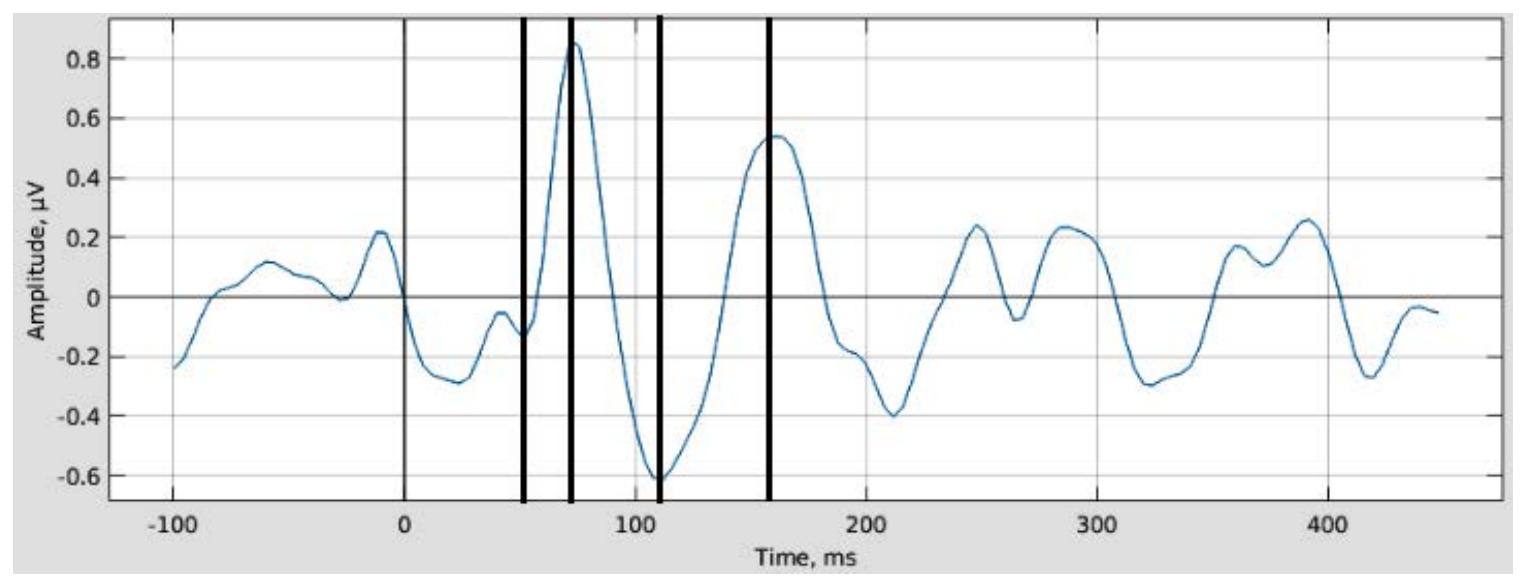

Figure 3. Averaged ERP response for a single participant with peaks and troughs identified with black vertical lines.

The components of interest were the P50, N100, and P200. Click 1 (S1) and Click 2 (S2) responses were averaged separately within each workload block to create component measures for each ERP within each workload condition. Both the amplitude of the component deflection and the peak onset latency of the components were compared for the younger and older groups, as well as sensory gating ratios. Clicks that were presented during the button press response for the visual task were excluded, due to the presence of motor-evoked potentials originating from the motor cortex.

Amplitude and latency effects were examined through $2 \times 3 \times 3$ mixed design ANOVAs, with a between-subject variable of age group (young adult and older adult), and within-subject variables of click type (S1, S2, or S2D for the deviant second click) and workload (baseline, easy, and hard). Latency was calculated as the time (milliseconds) to voltage peak following stimulus presentation for all triggers (S1, S2, and S2D). Sensory gating was investigated using mixed design $2 \times 2 \times 3$ ANOVAs, with a between-subject variable of age group, and within subjects variables of gating condition 
(standard gating condition as $\mathrm{S} 2 / \mathrm{S} 1$, and deviant gating condition asS2D/S1) and workload. Post-hoc pairwise contrasts were corrected with a Sidak correction. All tests and dependent variables were inspected to ensure that they did not deviate from a normal distribution.. 


\section{Chapter 4: Results}

First, results from the behavioural match-to-sample task are presented, followed by analyses of the EEG results from the PCOP task within each workload condition. $\mathrm{R}$ and SPSS were used to analyze the outcomes of the visual task data and subjective ratings of workload, while EEGLAB and SPSS were used to analyze the ERP data. To confirm that the assumptions of the mixed-design ANOVAs were met, distribution of residuals were tested with a Mauchly's test of sphericity, and Q-Q plots were examined.

\subsection{Match-to Task Dependent Variables}

Performance on the visual grid-matching task was examined with a 2 (Condition: easy vs. hard) x 2 (Age: younger vs. older adults) mixed design ANOVA, with age as the between-subjects factor. There was a main effect of condition, $F(1,28)=227.14, p<$ $.001, \eta_{\mathrm{p}}{ }^{2}=0.89$, such that participants successfully matched more trials in the easy $(M=$ $56.2, S E=0.54)$ than the hard condition $(M=43.7, S E=0.87), p<.001,95 \%$ CI $[10.8$, 14.2]. There was no main effect of age, but there was a significant age by condition interaction, $F(1,28)=8.8, p<.01, \eta_{\mathrm{p}}{ }^{2}=0.24$. As shown in Table 1 , age differences were 
only significant in the hard-workload condition, where young adults correctly matched more trials that older adults, $p=.017,95 \%$ CI $[0.84,7.96]$.

Table 1. Descriptive Results for the Number of Correctly Classified Grids ANOVA (maximum $=60$ )

\begin{tabular}{lccc}
\hline \multicolumn{1}{c}{ Condition } & Age Group & Number Correct & Standard Deviation \\
\hline Easy & Young Adult & 55.9 & 3.17 \\
& Older Adult & 56.5 & 2.75 \\
Hard & Young Adult & 45.9 & 4.67 \\
& Older Adult & 41.5 & 4.85 \\
\hline
\end{tabular}

A second 2x2 mixed design ANOVA using response time as the dependent variable revealed a main effect of condition, $F(1,28)=105.58, p<.001, \eta_{\mathrm{p}}{ }^{2}=0.79$, with longer latencies in the hard-workload $(M=1393, S E=55.72)$ than the easy-workload condition $(M=1044, S E=42.58)$. The main effect of age was marginally significant, $F(1,28)=4.14, p=.051, \eta_{\mathrm{p}}{ }^{2}=0.13$, with post-hoc pairwise comparisons revealing that older participants $(M=1509, S E=78.8)$ had significantly longer response times during the hard workload compared to younger participants $(M=1277, S E=78.8), p=.046$, 95\% CI $[4.07,460.64]$. Older participants $(M=1393, S E=55.72)$ did not significantly differ from younger participant response times during the easy workload $(M=1393, S E=$ 55.72), $p=.096,95 \%$ CI $[-27.67,321.21]$. Taken together, these results verify that the 
manipulation of task difficulty within the match-to-sample task produced behavioural differences (Figure 4).

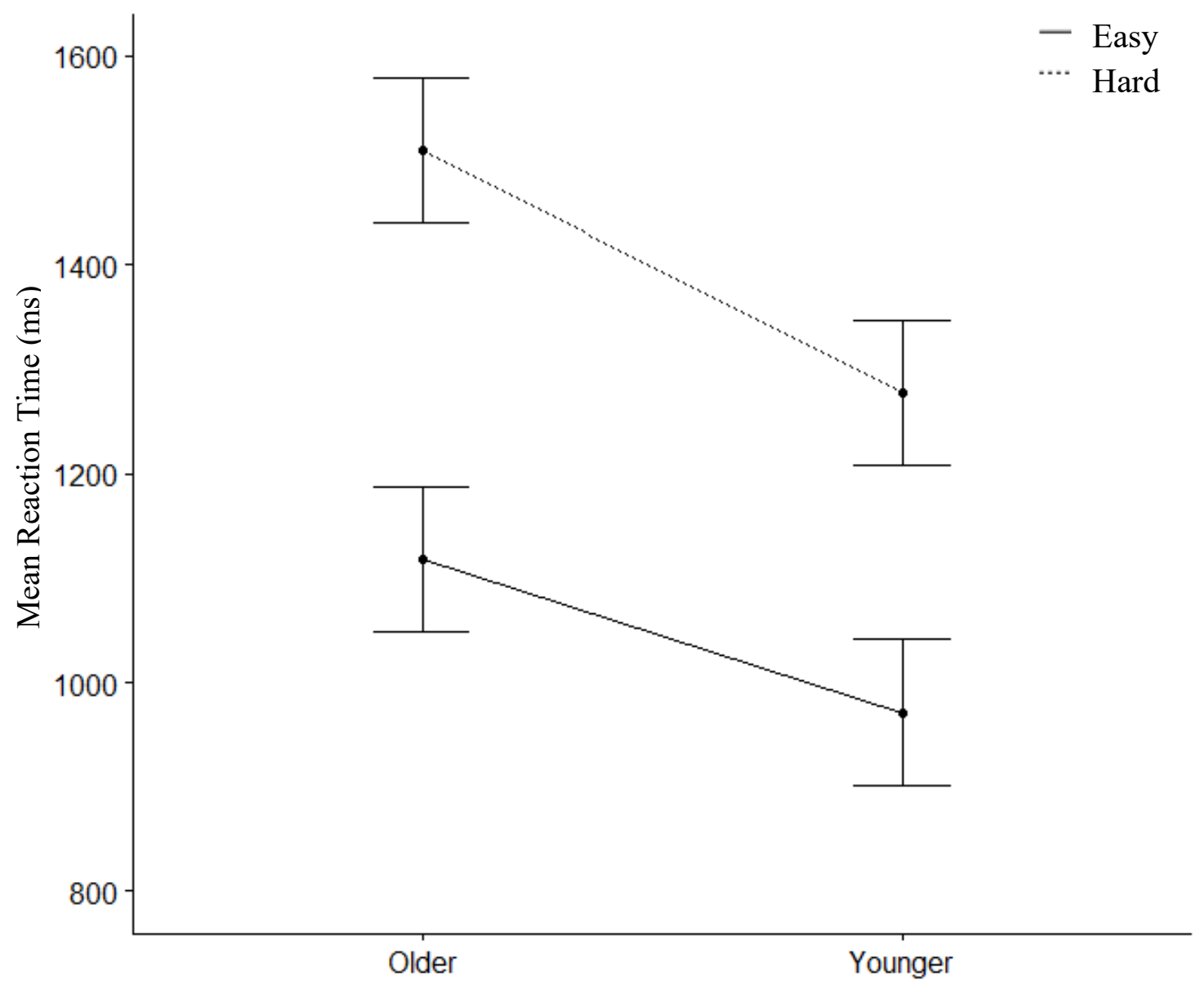

Figure 4. Mean reaction time for each age group, separated by workload condition.

\subsubsection{Subjective Workload Assessment}

To assess subjective workload, responses to the 5-point scale were averaged by workload condition. A mixed measures ANOVA with a between-subjects variable of age group and a within subjects variable of condition showed that participants rated the hard workload condition $(M=3.55, S D=0.63)$ as more difficult than the easy condition $(M=$ 
$1.73, S D=0.69), F(1,28)=180.8, p<.001$, but that ratings did not differ between age groups. There was no significant interaction present.

\subsection{ERP Results}

The following section presents the results from the EEG recordings for each of the three ERPs (P50, N100, and P200) within each workload (baseline, easy and hard) for each age group (younger and older adult). Amplitude and latency were analyzed using 3factor mixed design ANOVAs, with within subjects variables of workload and click type and age as the between subjects variable. Differences in sensory gating ratios were also analyzed using 3-factor mixed design ANOVAs, but with the within subject variables being workload and gating condition instead of click type. All violations of sphericity were non-significant unless otherwise stated. Confidence intervals are reported as $95 \%$ confidence intervals around mean differences. Error bars within all figures are represented as $95 \%$ confidence intervals calculated using the mean square error from the respective interaction effect (Campbell \& Thompson, 2012). For example, in the 3-level

plots, error bars were calculating using the mean square error from the 3-way interaction as indicated by SPSS. For this section, all means displayed within figures represent the estimated marginal means from the 3-way interaction. All p-values for pairwise comparisons were corrected using a Sidak correction.

\subsubsection{Paired-click validation}

To ensure that the classic paired-click effect for standard clicks was still present an examination of S1 and S2 amplitudes between age groups across workloads was conducted. Successful suppression of the second click would result in sensory gating ratios that are less than 1, with the amplitude of S2 divided by the amplitude of S1 (i.e., 
$\mathrm{S} 2 / \mathrm{S} 1)$. The Table 2 presents sample means for each age group within the baseline condition for S1 and S2 and their respective standard deviations. In all instances, except for the P50 for older adults, S2 amplitudes are less than S1 amplitudes, indicating suppression of the second standard click as per sensory gating. It is possible that the presentation of the deviant second click biased the amplitude of S2 for older participations, resulting in an inflated S2 amplitude. Future work using continuous EEG measures could investigate sensory gating ratios on a trial-by-trial basis to determine if older adults were biased towards the anticipatory nature of the deviant click.

Table 2. Mean S1 and S2 amplitudes (uV) during baseline for each ERP, separated by age.

\begin{tabular}{lccc}
\hline \multicolumn{1}{c}{ Age Group } & ERP & Mean S1 Amplitude (SD) & Mean S2 Amplitude (SD) \\
\hline Young & P50 & $.78(.57)$ & $.65(.34)$ \\
Older & & $.78(.45)$ & $.96(.44)$ \\
Young & $\mathrm{N} 100$ & $1.67(.73)$ & $.99(.67)$ \\
Older & & $1.77(.91)$ & $1.21(.78)$ \\
Young & $\mathrm{P} 200$ & $3.31(1.44)$ & $1.11(.86)$ \\
Older & & $2.37(1.04)$ & $1.67(.73)$ \\
\hline
\end{tabular}

\subsubsection{P50 Results}

\subsubsection{P50 Amplitude Results}

A 3-factor mixed design ANOVA (age by workload by click type) revealed no significant main effects of age, workload, or click type, as well as no significant interactions. However, as shown in Figure 5, there was a trending difference between younger and older participants in the hard workload condition, $p=.089,95 \%$ CI [-.057, 
.73], such that older participants had higher P50 amplitudes $(M=1.08, S E=0.15)$ for all click types compared to younger adults $(M=0.75, S E=0.12)$.

Examining the confidence intervals in Figure 5 suggests that difference between old and younger adults in the hard workload condition was only significant for S2. This indicates that the pre-attentive initial perception of the click, S1, is the same for both age groups, but that older adults have weaker suppression of the second click, S2, during hard workload.

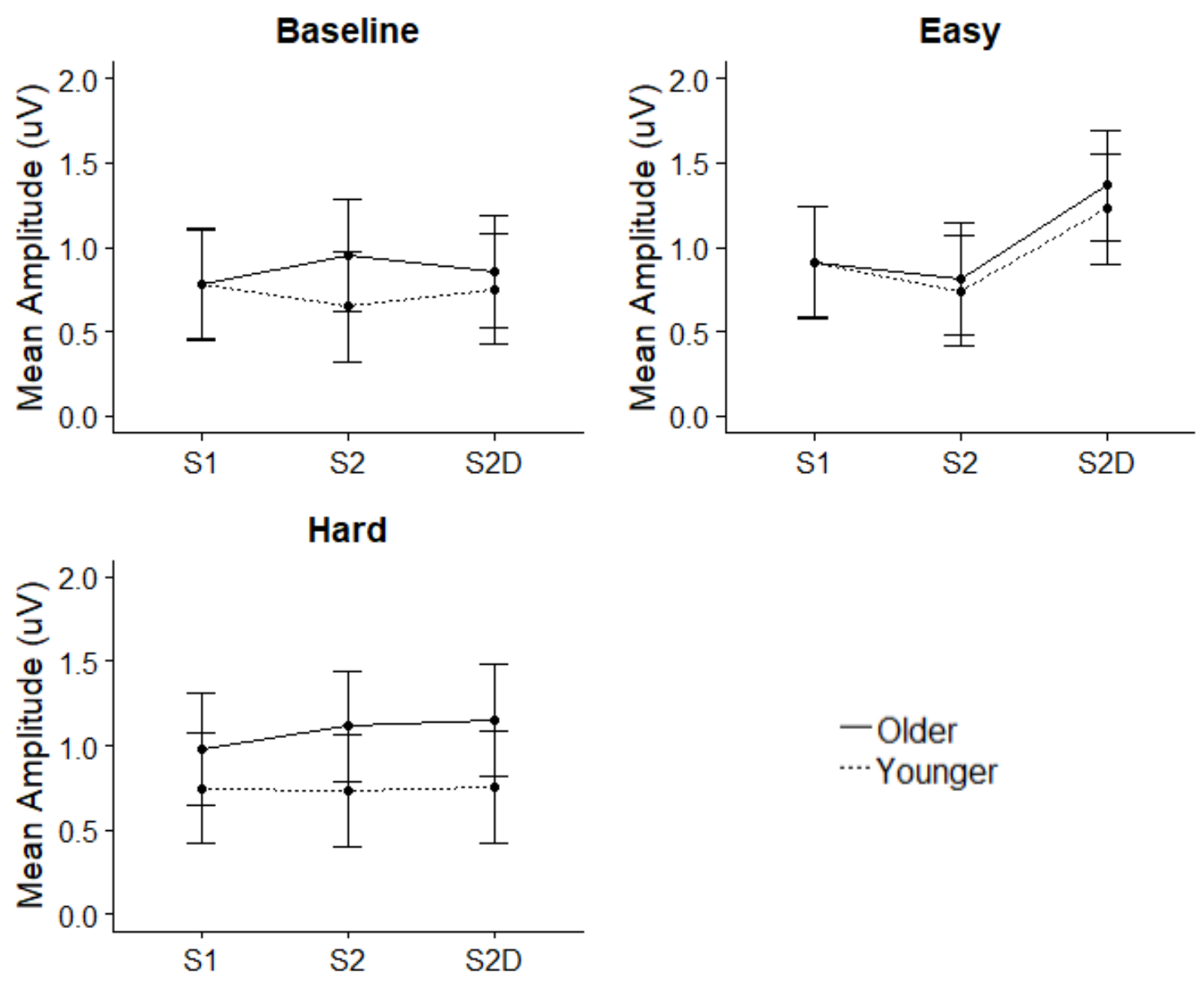

Figure 5. P50 amplitude by click type, separated by age group and workload. 


\subsubsection{P50 Latency Results}

A 3-factor mixed design ANOVA (age by workload by click type) revealed significant effect of workload on P50 latencies, $F(2,38)=4.79, p=.014, \eta_{\mathrm{p}}{ }^{2}=.20$, with longer latencies during the hard workload condition $(M=69.13, S E=1.80)$ compared to baseline $(M=61.18, S E=2.06), p<.01,95 \%$ CI [1.88 14.01]. Component latency is calculated as the time difference between stimulus presentation and the component peak. Latencies during the easy workload condition $(M=65.68, S E=2.06)$ were not statistically different from the hard or baseline conditions. No age significant main effects of click type or interactions were found. Examining pairwise comparisons between age group and click type revealed a trending significant comparison between younger and older participants, $p=.075,95 \%$ CI [-0.69 13.04], which was being driven by a significant difference when comparing age groups during baseline. Here, latencies for S1 were significantly larger for younger $(M=64.95, S E=2.54)$ compared to older adults $(M$ $=57.42, S E=3.24), p<.001,95 \%$ CI [7.1 24.06] (Figure 6). Although, examining the confidence interval bars suggests that this is not a strong effect. Regardless, this suggests 
that younger participants do not need to initiate the pre-attentive P50 as quickly as older adults. No other pairwise comparisons were significant.
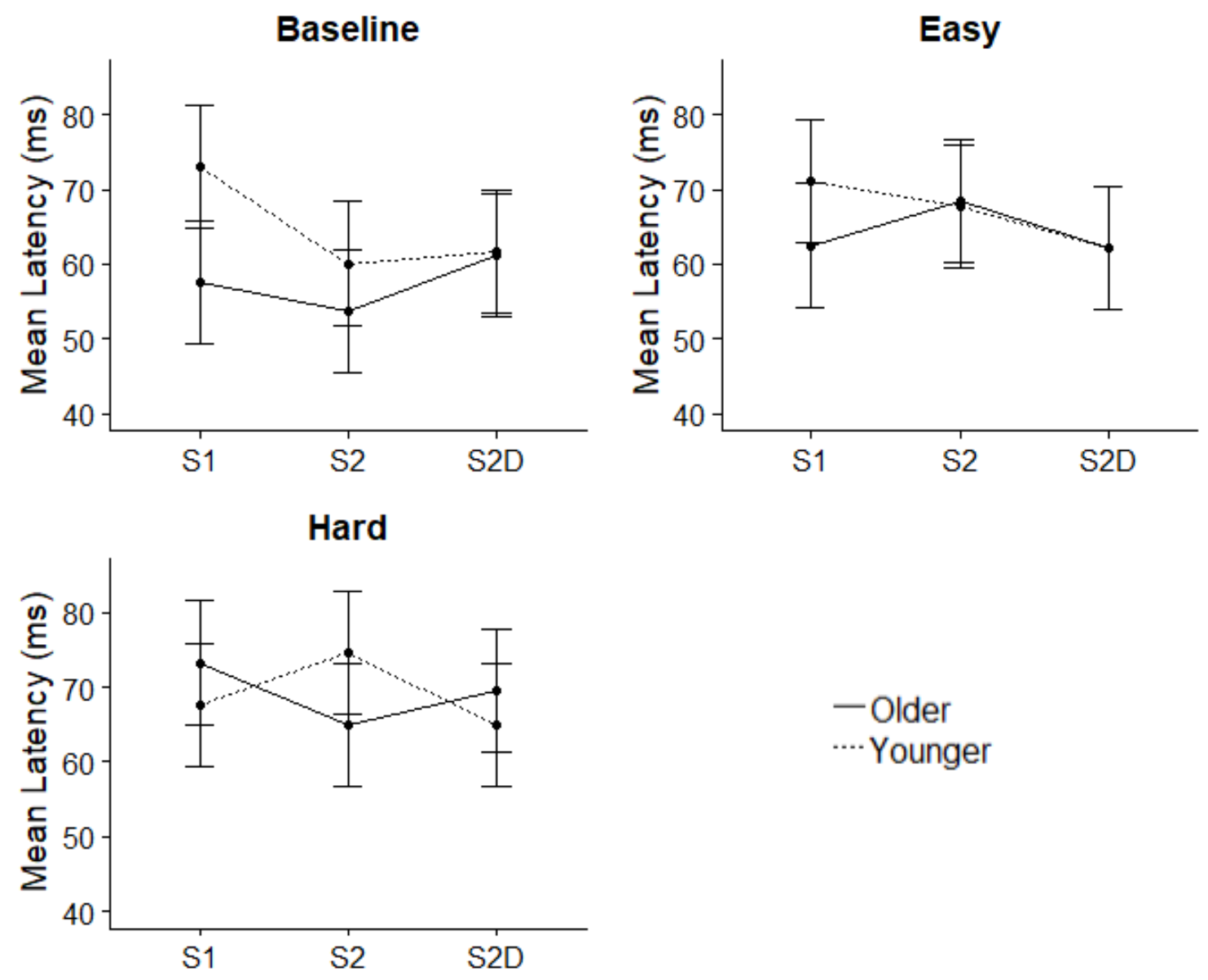

Figure 6. P50 latency by click type, separated by age group and workload.

\subsubsection{P50 Sensory Gating Ratios}

A 3-factor mixed design ANOVA (age by workload by gating condition) revealed a significant main effect of gating condition, $F(1,20)=6.36, p=.02, \eta_{\mathrm{p}}{ }^{2}=.24$, such that, deviant gating ratios $(M=1.58, S E=0.18)$ were significantly larger than standard gating ratios $(M=1.2, S E=0.077), p=.02,95 \% \mathrm{CI}[.07 .70]$ (Figure 7). Large deviant gating ratios compared to standard deviant ratios suggests that sensory gating is a dynamic 
mechanism, able to be influenced by unexpected stimuli when the traditional response is suppression. No other significant main effects or interactions were found.

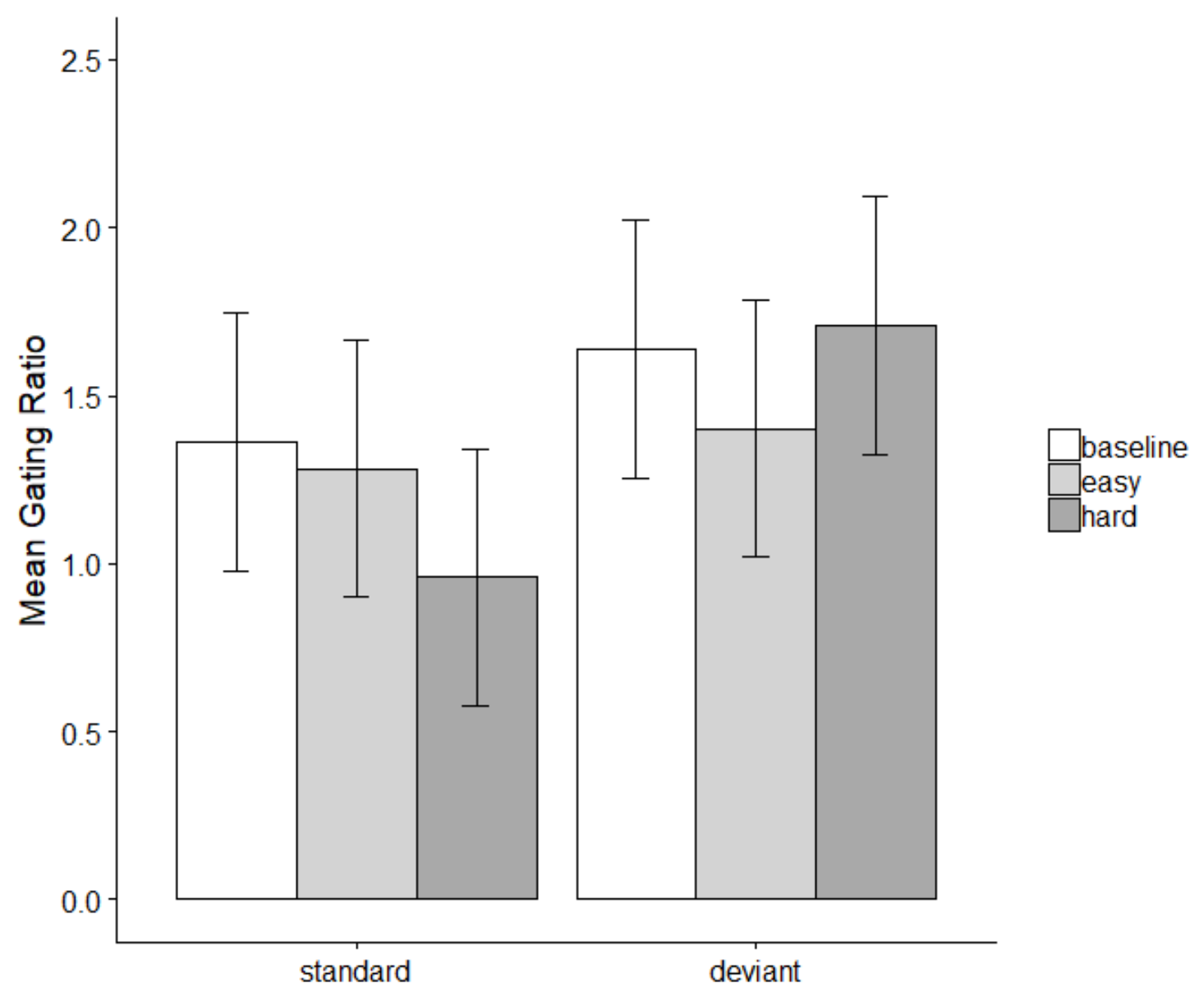

Figure 7. Mean P50 gating ratio by gating condition and workload.

Pairwise comparisons revealed marginal significance for standard gating ratios where older participants had larger P50 standard gating ratios $(M=1.36, S E=0.19)$ than younger adults $(M=1.04, S E=0.098), p=.05,95 \% \mathrm{CI}[0.0 .64]$, regardless of workload. 
Inspecting Figure 8 however reveals that this observation was only true during baseline and hard workload, but not during easy workload.

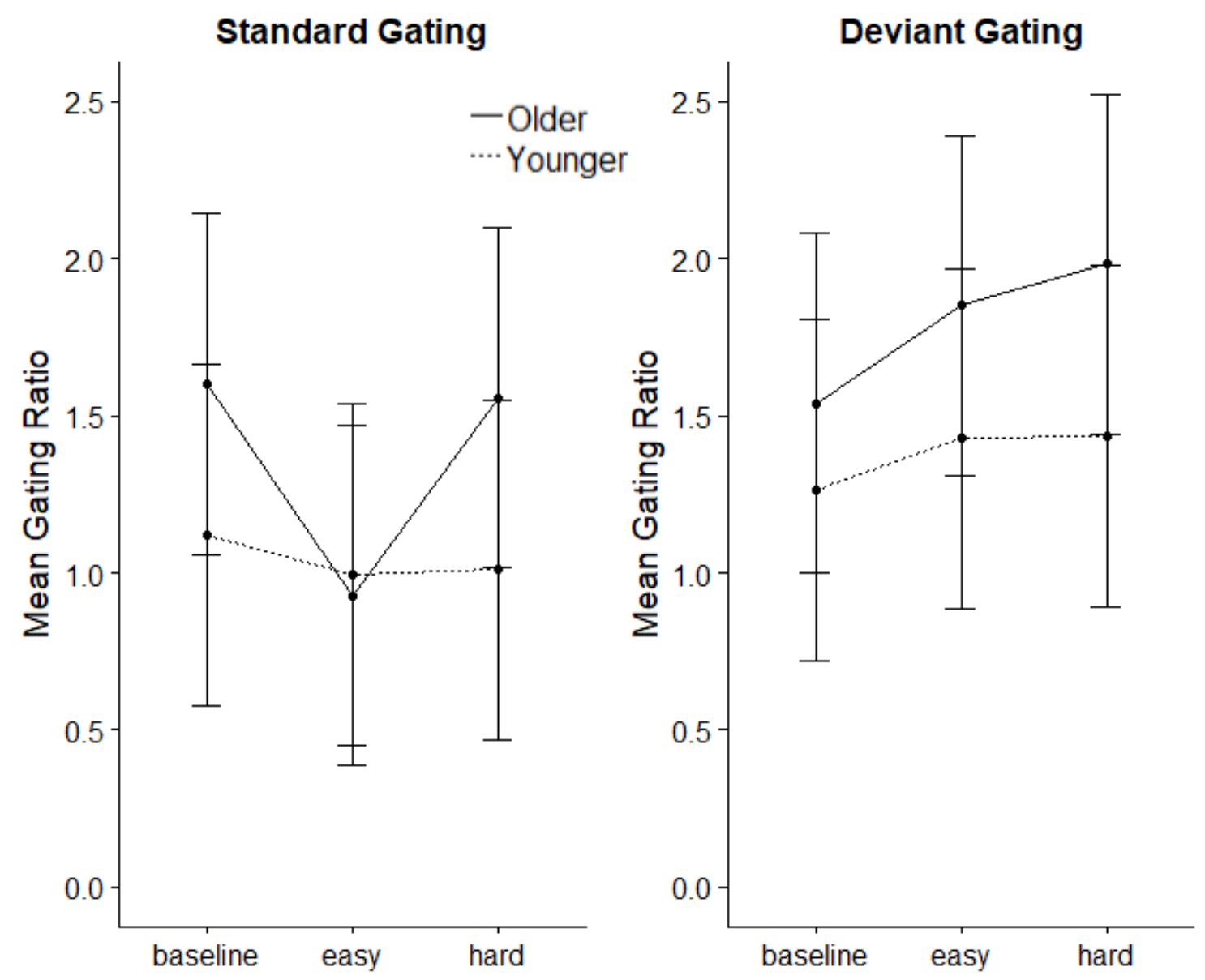

Figure 8. P50 gating ratio by workload, separated by age group and gating condition.

\subsubsection{P50 Summary}

In summary, deviant gating ratios at P50 were found to be larger than standard gating ratios. This result was marginally dependent on age, but not workload. Latencies during the hard workload condition were longer than baseline latencies for both age groups, and older adults tended to have larger P50 amplitudes compared to younger 
adults. Taken together, these results suggest that, even at the pre-attentive stage of processing, deviant information has the ability to influence sensory gating.

\subsubsection{N100 Results}

\subsubsection{N100 Amplitude Results}

An overall significant main effect of click type was present, such that S1 ampiltudes $(M=1.59, S E=0.18)$ were larger than $\mathrm{S} 2$ amplitudes $(M=1.12, S E=$ 0.087 ), $F(2,38)=7.72, p=.002, \eta_{\mathrm{p}}^{2}=.29$ (Figure 9). No significant main effects of workload or age group were present. There was a significant 3-way interaction of click type, workload, and age group, such that for the deviant second click in the easy


-... Younger

Figure 9. N100 amplitude by click type, separated by age group and workload. 
workload condition, older participants showed greater amplitudes $(M=2.28, S E=0.39)$ than younger participants $(M=1.24, S E=0.45), F(4,76)=4.04, p=.005, \eta_{\mathrm{p}}{ }^{2}=.18$. Larger S2D amplitudes for older adults suggests that suppression of the unexpected second click is weaker when older adults are focused on a visual task. All other comparisons were non-significant. There was a marginal trend for older participants to also have larger amplitude deflections for the standard second click in the hard workload condition $(M=1.38, S E=0.17)$ than younger participants $(M=0.78, S E=0.2), p=.094$, $95 \%$ CI [-.20 2.27].

\subsubsection{N100 Latency Results}

There were two significant main effects within the latency mixed design ANOVA: a main effect of click type, $F(2,38)=4.02, p=.026, \eta_{\mathrm{p}}{ }^{2}=.18$, with post-hoc tests revealing that $\mathrm{S} 1$ latencies were significantly longer $(M=105.69, S E=2.24)$ than S2 latencies $(M=97.3, S E=2.14), p<.01,95 \%$ CI [3.17 13.61] (Figure 10). This suggests that there may be an anticipatory element to perceiving the second click, with second click peaks occurring faster than first click peaks. 


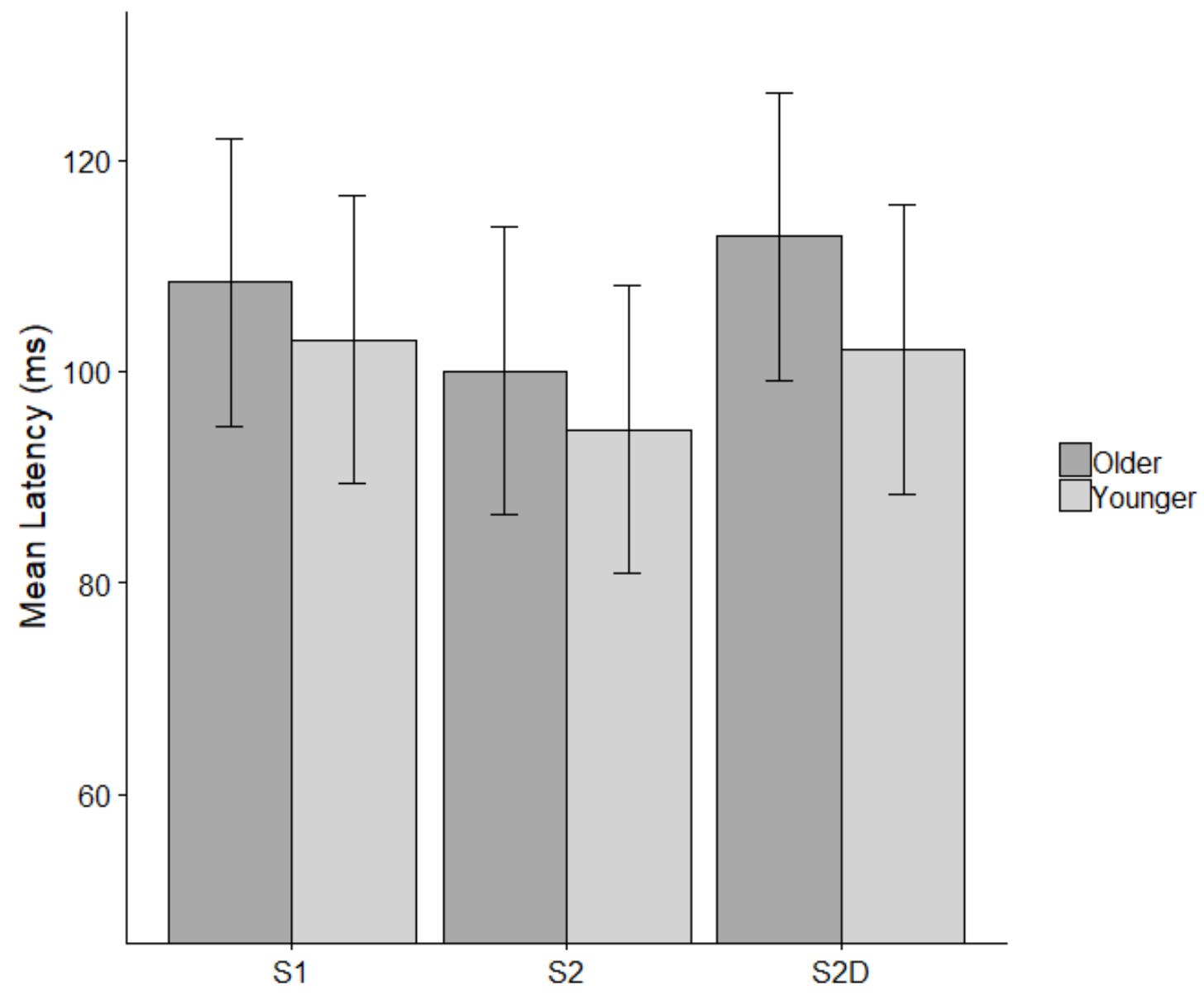

Figure 10. N100 latencies by click type and age group.

There was also a significant main effect of age, $F(1,19)=6.36, p=.026, \eta_{\mathrm{p}}^{2}=$ .25 , such that older adults had longer latencies $(M=107.09, S E=1.88)$ regardless of workload when compared to younger adults $(M=99.85, S E=2.17)$ for the N100, $p=$ $.021,95 \%$ CI [1.23 13.25] (Figure 11). This shows that, overall, peak amplitudes for the N100 occur later for older adults, suggesting that the attentive processing of the clicks is occurring later in time than younger adults. No significant interactions or main effects of workload were present. 
Baseline
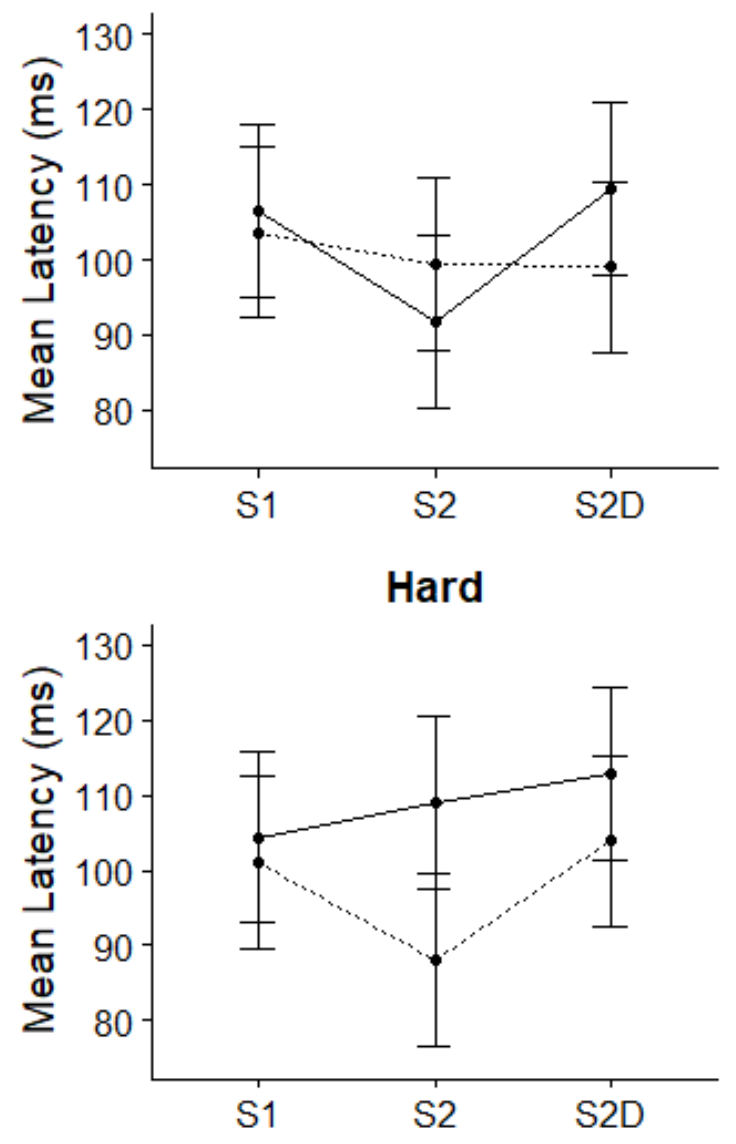

Easy

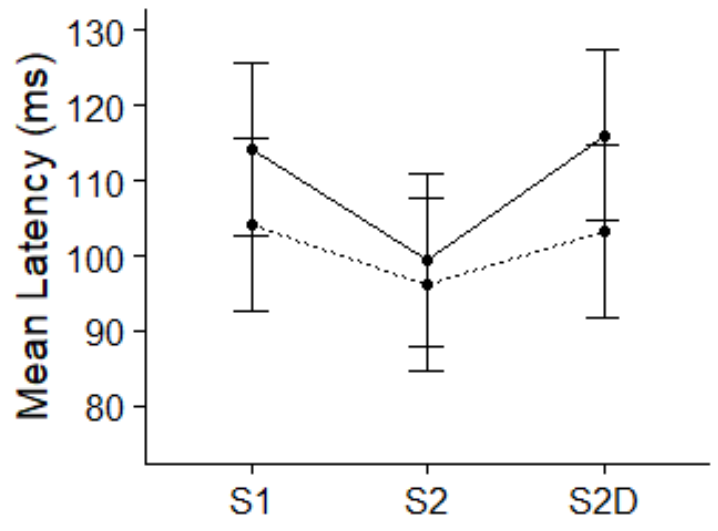

- Older

-... Younger

Figure 11. N100 latencies by click type, separated by age group and workload.

\subsubsection{N100 Sensory Gating Ratios}

No significant differences in sensory gating ratios between workload, age, or gating condition were found. However, the main effect of gating condition was trending, $F(1,22)=3.14, p=.09, \eta_{\mathrm{p}}^{2}=.13$, suggesting that deviant gating ratios $(M=1.24, S E=$ $0.11)$ are larger than standard gating ratios $(M=.96, S E=0.19)$ (Figure 12). Pairwise comparisons revealed a trending significant effect when comparing older $(M=1.10, S E=$ $0.31)$ and younger $(M=1.9, S E=0.17)$ deviant gating ratios during the hard workload condition, suggesting that younger adults exhibited weaker sensory gating than their older 
counterparts for the N100, $p=.087,95 \%$ CI [-.13 1.84] (Figure 12). This suggests that although younger participant N100 amplitudes were smaller overall, sensory gating ratios suggest that this does not translate to stronger sensory gating. Examining the baseline standard gating ratio condition, younger adults appear to have weaker N100 gating. This suggests that differences between age groups depends on the ERP being examined. No pairwise comparisons were significant.

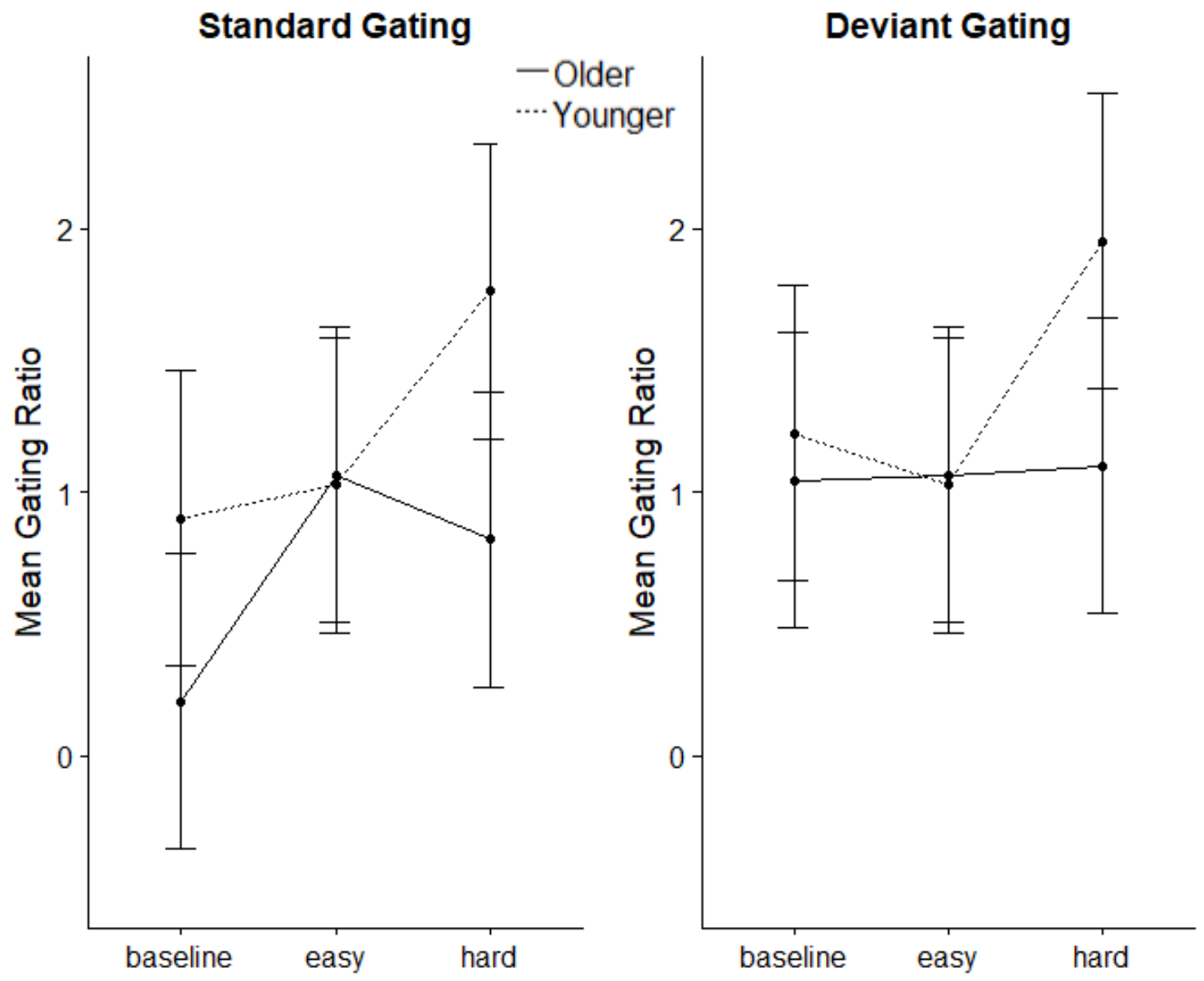

Figure 12. N100 gating ratio by workload, separated by age group and gating condition.

\subsubsection{N100 Summary}

Older adults were found to have longer N100 latencies, regardless of click type and workload, than younger adults. Regardless of age or workload, S1 latencies and 
amplitudes were found to be longer and greater, respectively, than latencies and amplitudes for S2. Larger S1 amplitudes compared to S2 is indicative of the sensory gating effect. Although no differences were significant when examining gating conditions, there was a trend towards larger deviant gating ratios than standard gating ratios, which is similar to the P50 result. In addition, younger participants were found to have weaker standard gating ratios during hard workload. These results suggest that the N100 is also sensitive to sensory gating, that the magnitude of gating in part depends on workload, and that younger adults appear to gate less during high workload when examining this ERP.

\subsubsection{P200 Results}

\subsubsection{P200 Amplitude Results}

A significant main effect of click type was found, $F(2,46)=21.51, p<.001, \eta_{\mathrm{p}}{ }^{2}=$ .48 , such that $\mathrm{S} 2$ amplitudes $(M=1.50, S E=0.17)$ were found to be significantly smaller than S2D amplitudes $(M=2.21, S E=0.21), p<.001,95 \% \mathrm{CI}[.7281 .43]$, and S1 amplitudes $(M=2.58, S E=0.25), p<.01,95 \%$ CI [.255 1.17]. S1 amplitudes were not significantly different from S2D amplitudes. This means that the neurological response to the second deviant click had a similar amplitude to the first click, and suggests that deviant stimuli were able to overcome the typical suppression response associated with the second click. There was also a significant age group by click type interaction, $F(2,46)$ $=8.66, \mathrm{p}<.001, \eta_{\mathrm{p}}{ }^{2}=.27$, but the main effect of age group was not significant. Post-hoc pairwise comparisons between age and click type revealed that age groups differed when 
comparing S1 amplitudes only, $p=.039,95 \%$ CI [.06 2.16], such that younger participants had larger $\mathrm{S} 1$ amplitudes $(M=3.13, S E=0.37)$ than older adults regardless


Figure 13. P200 amplitude by click type, separated by age group and workload.

of workload $(M=2.02, S E=0.35)$. This demonstrates that older adults are at a disadvantage when the gating ratio is calculated because their initial response to the first click is already significantly attenuated. However, this result is better explained by examining the significant 3-way interaction. 
A significant 3-way interaction between click type, workload, and age group was also found, $F(4,92)=2.99, p=.023, \eta_{\mathrm{p}}^{2}=.16$. Post-hoc pairwise comparisons reveal that the age difference between S1 amplitudes only occurred during the easy workload condition, where younger participants have significantly larger P200 S1 amplitudes ( $M=$ 3.34, $S E=0.42)$ than older adults $(M=1.69, S E=0.41), p=.01,95 \%$ CI $[.442 .86]$ (Figure 13). All other comparisons were non-significant, indicating that younger and older adults did not differ when comparing S2 and S2D amplitudes within the separate workload conditions. However, there was a trending significant result when comparing S1 amplitudes between age groups during the baseline condition, $p=.074,95 \%$ CI [-.097 1.98], indicating a similar observation to the easy workload S1 amplitudes with younger adults having larger $\mathrm{S} 1$ amplitudes $(M=3.31, S E=0.36)$ than older adults $(M=2.37, S E$ $=0.35)$. No other pairwise comparisons were significant.

\subsubsection{P200 Latency Results}

There was a significant main effect of click type, $F(2,40)=7.27, p<.01, \eta_{\mathrm{p}}{ }^{2}=$ .28 , with $\mathrm{S} 1$ latencies $(M=178.91, S E=2.87)$ were significantly longer than S2 latencies $(M=165.35, S E=3.08), p<.01,95 \%$ CI [3.07 24.04] (Figure 14). Latency differences between S2 and S2D and S1 and S2D were not significantly different from each other. No other main effects or interactions were significant. 


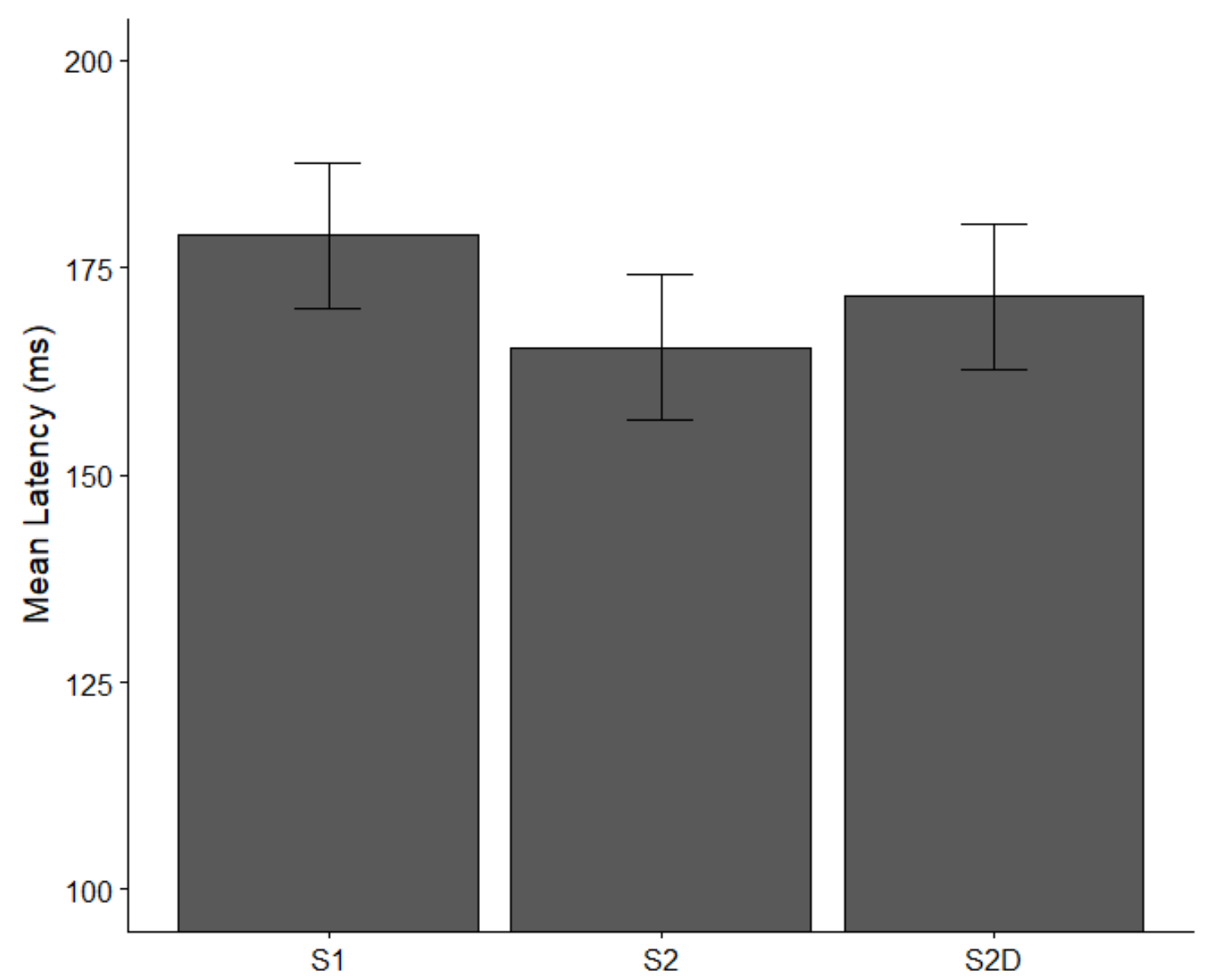

Figure 14. P200 latencies by click type.

However, there was a marginal result observed for the workload by click type interaction, $F(4,80)=2.37, p=.06, \eta_{\mathrm{p}}^{2}=.11$, with a marginal difference between S2D amplitudes when comparing baseline and easy workload, suggesting longer latencies in the easy $(M=182.29, S E=5.36)$ than the baseline condition $(M=164.15, S E=5.27), p$ $=.086,95 \% \mathrm{CI}[-2.0438 .33]$ (Figure 15). No other pairwise comparisons were significant. 
Baseline
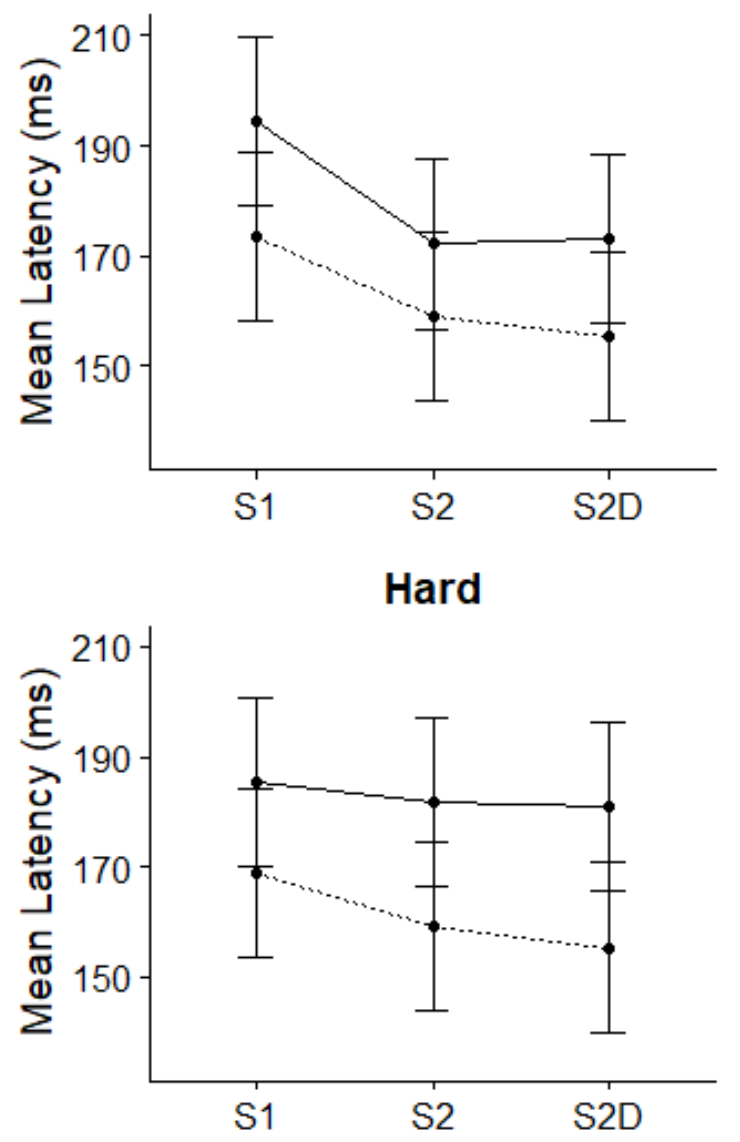

Easy



- Older

-... Younger

Figure 15. P200 latencies by click type, separated by age group and workload.

\subsubsection{P200 Sensory Gating Ratios}

There was an overall significant main effect of gating condition, $F(1,23)=16.96$, $p<.001, \eta_{\mathrm{p}}{ }^{2}=.42$, such that gating during the deviant condition $(M=1.04, S E=0.10)$ was significantly weaker than during the standard condition $(M=0.64, S E=0.043), p<$ $.001,95 \% \mathrm{CI}[0.20 .6]$. Similar to the trend observed in the N100 and significant effect of gating condition in the P50, these results provide evidence that sensory gating is a dynamic mechanism, and that the degree of gating varies across ERPs. There was a 
marginal main effect of workload, such that gating was stronger in the baseline $(M=$ $0.68, S E=0.08)$ compared to the easy $(M=0.93, S E=0.081)$ and hard $(M=0.90, S E=$ $0.084)$ conditions, $F(1,23)=3.87, p=.061, \eta_{\mathrm{p}}{ }^{2}=.11$.

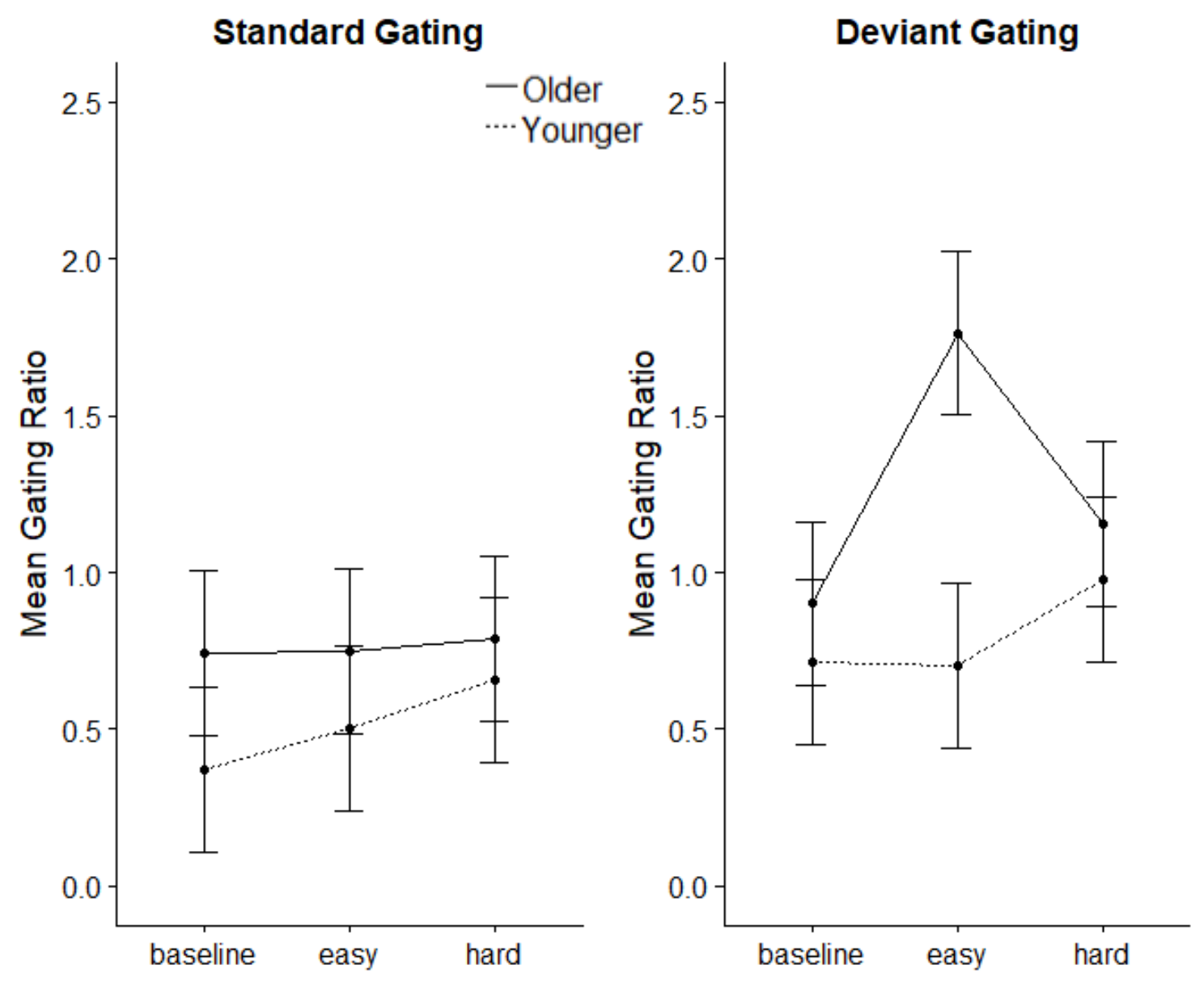

Figure 16. P200 gating ratios by workload, separated by age group and gating condition.

There was a significant main effect of age, such that older adults had larger gating ratios $(M=1.02, S E=0.08)$ compared to younger adults overall $(M=0.65, S E=0.08)$, $F(1,23)=10.774, p=.003, \eta_{\mathrm{p}}{ }^{2}=.319$. Observing larger gating ratios for older adults provides evidence that sensory gating is impacted by age. However, results from the 
P200 amplitude provide evidence that this difference in gating ratios might be driven by the attenuated initial response seen in older adults, and not the inability to suppress the response to the second click. There was also a significant 3-way interaction between gating condition, workload, and age, $F(2,46)=4.15, p=.023, \eta_{\mathrm{p}}{ }^{2}=.15$ (Figure 16). Posthoc pairwise comparisons demonstrated that older adults had larger standard gating ratios $(M=0.74, S E=0.09)$ than younger adults during the baseline condition $(M=0.37, S E=$ $0.09), p<.01,95 \% \mathrm{CI}[.12 .63]$, as well as larger deviant gating ratios $(M=1.76, S E=$ $0.20)$ than younger adults during easy workload $(M=0.71, S E=0.21), p<.001,95 \% \mathrm{CI}$ $[.471 .65]$

\subsubsection{P200 Summary}

Older adults had weaker gating ratios than younger adults. Overall, deviant gating ratios were larger than standard gating ratios, indicating weaker gating for deviant clicks, and larger S2D amplitudes than S2 amplitudes. A significant 3-way interaction revealed that older adults gated less than younger adults during baseline for standard clicks, and much less than younger adults during easy workload for deviant clicks. This finding is further justified by examining the separation between the $95 \%$ confidence interval bars in Figure 16. Although older adults were found to gate less overall, their S1 amplitudes are significantly smaller than younger adults, indicating a disadvantage for the first click when calculating the sensory gating ratio. Post-hoc tests revealed that S1 disadvantages 
for older adults were only significant during the easy workload. S1 latencies were found to be significantly longer than S2 latencies, which was also found for the N100 data.

\subsection{Correlations between Sensory Gating Ratios and Match-To Performance}

To help further determine whether standard sensory gating is a general purpose mechanism or sensitive to changes in workload, a correlational analysis was conducted between the standard sensory gating ratios for each of the three ERPs from each condition (P50: baseline, easy, hard; N100: baseline, easy, hard; P200: baseline, easy, hard) and the number of correctly matched grids (easy and hard workload) to create an 11x11 correlation matrix. Results were first collapsed across age groups, and then separated into older and younger adults.

Collapsed across age groups, there was a significant positive correlation between P50 gating and N100 gating in both the baseline, $r=0.47, p=.01$, and during the easy condition, $r=0.50, p<.01$. Having a smaller sensory gating ratio at the level of the P50 is therefore associated with smaller N100 gating ratios, indicating better filtering of the irrelevant second click. When separated by age groups, this pattern of results is only seen for the older adults, such that larger sensory gating ratios the P50 are associated with larger N100 gating ratios during the easy workload, $\mathrm{r}=0.61, \mathrm{p}=.028$, and baseline conditions, $\mathrm{r}=0.67, \mathrm{p}<.01$. No significant correlations between P50 or N100 gating ratios and P200 gating were found during any of the conditions.

Smaller N100 baseline gating ratios were also found to be associated with better performance during the hard workload condition, $r=-0.41, p=.036$. This correlation was not found when age groups were separated. A significant correlation between the number of correct responses during the easy workload condition and the P50 gating ratio during 
hard workload, $r=-0.41, p=.036$, was present was collapsed across age groups, but only present in the young adult group after separation, $r=-0.70, p<.01$. When separated by age, there were no significant correlations between gating ratios and match-to task performance for older adults.

Correlations between reaction time for the two workload conditions was also examined. When collapsed across age groups, there were significant positive correlations between reaction time within hard workload and P200 baseline gating, $r=0.38, p=.045$, and gating during the hard workload condition, $r=0.44, p=.022$, indicating that weaker gating was associated with longer reaction times. However, when separated by age group, these correlations were no longer significant. This result is could be due to the distribution of $\mathrm{P} 200$ gating ratios and reaction times being split between younger and older participants, and when collapsed across age, these two age groups form the two sides of each dependent variable distribution.

In sum, although sensory gating measures were not found to correlate with performance outcomes, weaker sensory gating during the pre-attentive stage was associated with weaker sensory gating during the N100 for older adults. This suggests that degree of gating can be carried through subsequent ERPs for older adults.

\subsection{Summary Table}

The following table summarizes the significant effects seen within each ERP and dependent variable measure. If no significant effect was present, then "N/A" is shown. Trending results discussed in text are not included. 
Table 3. Summarized significant effects for each ERP dependent variable.

\begin{tabular}{|c|c|c|c|}
\hline & P50 & N100 & P200 \\
\hline Amplitude & $\begin{array}{l}\text { Weaker suppression of } \\
\text { S2 for older adults } \\
\text { during hard workload. }\end{array}$ & $\begin{array}{l}\text { Older adults had greater } \\
\text { S2 deflection during the } \\
\text { hard workload } \\
\text { condition. } \\
\text { S1 amplitudes were } \\
\text { larger than S2 } \\
\text { amplitudes, regardless } \\
\text { of age and workload. }\end{array}$ & $\begin{array}{l}\text { S2 amplitudes were } \\
\text { smaller than S2D and } \\
\text { S1 amplitudes, but S1 } \\
\text { amplitudes were not } \\
\text { significantly different } \\
\text { from S2D amplitudes. } \\
\text { Older adults had } \\
\text { smaller S1 } \\
\text { amplitudes, } \\
\text { regardless of } \\
\text { workload, but this } \\
\text { was only significant } \\
\text { within easy workload. }\end{array}$ \\
\hline Latency & $\begin{array}{l}\text { Longer latencies } \\
\text { overall during hard } \\
\text { workload compared to } \\
\text { baseline, regardless of } \\
\text { age. } \\
\text { Younger participants } \\
\text { had longer latencies for } \\
\text { S1 during baseline. }\end{array}$ & $\begin{array}{l}\text { Older adults had longer } \\
\text { latencies, regardless of } \\
\text { workload and click } \\
\text { type. } \\
\text { S1 latencies were } \\
\text { longer than S2 } \\
\text { latencies. }\end{array}$ & $\begin{array}{l}\text { S1 latencies were } \\
\text { longer than S2 } \\
\text { latencies. }\end{array}$ \\
\hline $\begin{array}{l}\text { Sensory } \\
\text { Gating }\end{array}$ & $\begin{array}{l}\text { Weaker gating for } \\
\text { deviant gating } \\
\text { condition compared to } \\
\text { standard gating } \\
\text { condition. }\end{array}$ & $\mathrm{N} / \mathrm{A}$ & $\begin{array}{l}\text { Older adults had } \\
\text { weaker sensory } \\
\text { gating, regardless of } \\
\text { gating condition and } \\
\text { workload. } \\
\text { Weaker gating for } \\
\text { deviant gating } \\
\text { condition compared } \\
\text { to standard gating } \\
\text { condition. } \\
\text { Older adults had } \\
\text { larger standard gating } \\
\text { ratios during the } \\
\text { baseline condition, as } \\
\text { well as larger deviant } \\
\text { gating ratios during } \\
\text { easy workload. }\end{array}$ \\
\hline
\end{tabular}




\section{Chapter 5: Discussion}

The present thesis investigated the impact of age on the sensory gating mechanisms across pre-attentive and attentive stages of auditory processing, and used a newly adapted paradigm to test the hypothesis that deviant information is able to surpass the mechanism of sensory gating.

\subsection{Age influenced sensory gating ratios during attentive stages of processing}

The ability of deviant, or unexpected, information to redirect our attention is a crucial neurological process that changes how information is processed in the brain. Previous research has defined sensory gating as the ability to suppress neurological responses to repetitive information. However, research investigating the influence that age has on the mechanism of sensory gating is sparse, with mixed findings across the $\mathrm{P} 50, \mathrm{~N} 100$, and $\mathrm{P} 200$ gating ratios.

In the present research, younger and older adults were found to differ in their sensory gating ratios at $\mathrm{P} 200$. This difference was primarily found within the easy workload and baseline conditions. Larger sensory gating ratios within the standard gating condition are indicative of a reduced sensory gating mechanism, allowing irrelevant information to "leak" into higher order cognitive functions such as allocation of attention (Lijffijt et al., 2009). This interpretation makes sense when only considering the sensory gating ratio of the $\mathrm{P} 200$. But when one then considers how this ratio is highly dependent on the response amplitude to the first click, it is apparent that the differences between age groups are not only differences in ratio, but differences in how each group responds to the first auditory stimuli. Older participants had significantly smaller P200 amplitudes in response to the first click, whereas their responses to the second standard click do not 
differ in amplitude from their younger counterparts. Following this interpretation, the reason older and young adults differ in their sensory gating ratios is not because the response to the second click is larger, but because the initial response is smaller in older participants. That is, weaker gating was observed within the P200 for older adults because the initial neurological response to auditory stimuli is reduced. Acknowledging amplitude differences rather than only the calculated gating ratio allows for a more accurate interpretation of how age influences gating ratios.

Results from this thesis demonstrate that, at least in part, sensory gating for ERPs related to attentive processing are sensitive to changes in workload. The majority of previous research investigating sensory gating stresses the importance of a static, passive environment to test sensory gating (Gjini, Burroughs, \& Boutros, 2011). However, our day-to-day environment is neither passive nor static. Understanding how sensory gating works within a more complex environment provides evidence for how the brain is able to utilize computational mechanisms to enhance performance. While previous research has found age differences in N100 gating, participants were sitting in a highly control and quiet environment, not completing a visual task (Gmehlin et al., 2011). Results from the discussed work demonstrate that while immersed in an environment that requires selective attention, age differences are only seen when examining the P200. This could demonstrate that, in part, sensory gating is sensitive to changes in workload.

Despite age and workload differences in sensory gating, ratios were not correlated with performance on the match-to task. But, P50 gating and N100 gating during the baseline and easy workload conditions were found to be correlated. Although the size of the P50 and N100 gating ratios were not related to age, a significant correlation between 
P50 and N100 gating for older adults suggests a functional overlap, and that this association weakens during more attentive stages at the P200. Observing age differences in P200 amplitude and sensory gating with no changes in visual task performance or reaction times suggests that older and younger adults could be using separate neurological strategies during attentive processing stages to succeed at the visual task, at least when workload is moderate. Observing different age outcomes depending on workload has been proposed by theoretical models, such as the hemispheric asymmetry reduction in older adults (HAROLD) (Cabeza, 2002) and the compensation-related utilization of neural circuits hypothesis (CRUNCH) (Reuter-Lorenz \& Cappell, 2008), which attempt to explain how workload influences neural recruitment for aging adults during various levels of workload. Research findings from this study support the CRUNCH hypothesis, in that during scenarios where workload must be managed, older adults may recruit additional neurological resources as a compensatory mechanism. However, it was demonstrated that this over-activation quickly reaches a threshold during easy workload tasks (Causse, et al., 2019). The CRUNCH model predicts that as task load increases, older adults compensate through over-activation during lower workloads of activity relative to younger adults. Older adults then experience an under-activation during hard workload due to quickly reaching a resource ceiling (Causse, et al., 2019). Observing attenuated responses to auditory information during easy workload for older adults suggests that older adults could be over-recruiting neurological resources in order to perform well on the visual task, which leaves fewer processing resources for auditory information; hence the observed differences in P200 sensory gating between age groups and longer response times during hard workload for older adults. As workload further 
increased, neural recruitment strategies for older adults may have changed as a result of reaching a resource ceiling, leading to a reduction in age differences with respect to ERP amplitude differences. Because only a single electrode site was examined (i.e., $\mathrm{Cz}$, as it typically used for paired-click paradigms), changes in cortical recruitment as a result of age cannot be directly answered with the presented analyses.

However, ERPs are not modular occurrences. Event-related potentials are an averaged response, summed across hundreds of trials and, in the case of this study, investigated at a single point (i.e., Cz). Results from this study are similar to other current research findings, in that workload has an impact on the amplitude and latency of neurological responses and that this effect is more pronounced with age. However, the goal of this thesis was to further investigate the potential cognitive mechanisms that lead to these observed deficits. Although auditory evoked ERPs are a neurological response to an auditory event, the brain's response provides clues as to what else is happening in the brain. A reduced ERP component amplitude is not a result of a reduction in stimulus volume, or not perceiving a given stimulus. Reductions in amplitude in response to an auditory event provide us with evidence that fewer neurological resources are available to process the higher order, attentive integration of the auditory stimulus because similar brain areas are being recruited for other tasks. When completing tasks that demand management of attentional resources and selective attention, research has demonstrated that older adults tend to over-recruit neurological resources within their prefrontal cortices (Causse, Chua, Remy, 2019; Piefke, Onur, \& Fink, 2012).

A reduced sensory gating ratio within the easy workload condition for older adults demonstrates that an increase in workload not only leads to an attenuated response in 
older adults, but a decrease in the effectiveness of the brain to filter out irrelevant information. In sum, age differences in amplitude and sensory gating as a result of workload are not linear, and these differences could signify that older adults recruit different brain areas as task difficulty increases. Traditional filtering models like the bottleneck theory from Broadbent would predict that because participants are completing a visual primary task, the auditory clicks are left unattended. Broadbent's thought process would suggest that neurological responses should not change with workload; attention should be solely focused on the visual task, resulting in high and equal sensory gating across auditory processing stages. However, because workload differences were observed, more specifically during the attentive stages of auditory processing, results suggest that older and younger adults don't differ in their pre-attentive processing of stimuli, but do differ in their sensory gating abilities during stages that require attentive processes like selective attention.

\subsection{Impact of deviant information in sensory gating}

Classic sensory gating research involves two identical clicks, paired close in time, with the second click conceptualized as redundant information. The paired-click paradigm provides a measure for how efficiently the brain can filter out irrelevant information as a means for enhancing efficiency. However, our world is made up of both redundant and salient stimuli. To investigate whether sensory gating is a dynamic mechanism, the second click was replaced with a deviant click to see if unexpected stimuli could overcome the brain's traditional suppression response during the sensory gating paradigm. 
Overall, the standard sensory gating condition provided validation for the classic paired click task, with the amplitude of the second click being attenuated during the baseline condition. The modified PCOP task extended these findings in demonstrating that deviant stimuli were able to surpass the traditional mechanism of sensory gating within the N100 and P200 ERPs across various workloads within this study. Differences in gating ratios during standard and deviant conditions shows that sensory gating, as measured in the paired click paradigm, and detection of stimulus changes, as measured in the oddball paradigm, both occur in the modified PCOP paradigm. The attenuated response to the second click recovers when the click is deviant, showing that low-level processing mechanisms are continuously monitoring sensory information Larger sensory gating ratios within the $\mathrm{N} 100$ and $\mathrm{P} 200$ for deviant information may provide clues as to how attention is allocated within different workload environments. For example, deviant sensory gating ratios were found to be significantly higher in the easy workload condition for the P200, but not the N100. Differences in gating ratios during attentive stages could suggest that the P200 gating ratio is more sensitive to changes in workload than the N100 ratio. Further, older participants had weaker gating in response to the deviant second click compared to the younger participants. By not attenuating their response to the second deviant click, older participants were able to overcome the mechanism of sensory gating more easily compared to the younger participants.

Classically, sensory gating is said to be observed when the ratio of the second click over the first is less than one, indicating that the neurological response to the identical second click has been attenuated. Investigating sensory gating with an oddball click in place of the second click was a new research paradigm, and a priori, it was 
challenging to predict the magnitude of the effect that this would have. Results from this study show that the deviant clicks resulted in sensory gating values greater than one, indicating no suppression at all for the deviant click. Although it can be thought of as very weak gating, it is also valid to assume that lack of efficient gating is an indication of facilitation of processing. Therefore, when the stimuli were unexpected, gating was not present, which resulted in the second click being processed to a greater extent compared to the identical second clicks.

Results from the both the standard and deviant sensory gating conditions could be an indication of how sensory gating is slightly different for each ERP. Differences between standard and deviant gating ratios during the attentive stages of auditory processing indicate that sensory gating is a dynamic mechanism, both in the sense that it is sensitive to changes in workload and stimulus properties. Similar to finding age-related differences during P200 gating, these results further support the notion that older and younger adults do not differ in their pre-attentive, feature recognition responses to the PCOP task, but do differ in their neurological processing of stimuli during attentive stages. 


\section{Chapter 6: Conclusion}

The presented research supports the initial hypothesis that deviant clicks would overcome the mechanism of sensory gating, indicating that sensory gating is a dynamic mechanism. It was also found that sensory gating ratios were sensitive to changes in workload, but that gating ratios varied both by age, and whether the second click was identical or deviant from the initial click. For older adults, weaker P200 sensory gating ratios came as a result of an attenuated response to the first click, rather than a decrease in response to the second click. The observation that effects of age were seen in the P200 suggests that weaker sensory gating for older adults has more to do with the allocation of attention, and less with the pre-attentive perception of auditory stimuli. This conclusion is in line with research supporting a reduction in executive function for older adults. Replacing the second click with an oddball click created a novel paradigm, and may be useful for testing how allocation of attention to auditory tasks functions when individuals are in a complex environment that requires their attention to be focused on a separate cross-modal, primary task.

Using oddball auditory stimuli could be a useful tool for designing alarms that would facilitate detection of relevant stimuli for older adults in complex auditory environments, like flying an aircraft. The purpose of this study went beyond that of investigating sensory gating, and was designed with the idea that auditory alarms could be an efficient way to help mitigate age-related effects in auditory attention. Finding that oddball stimuli were able to surpass the gating mechanism for older adults was an initial 
step in stimulating further studies that would investigate how built in neurological mechanisms can assist older general aviation pilots in a safer flying experience.

\subsection{Limitations}

There are three limitations identified within this research that are worth highlighting. First, because only a single electrode site was used (i.e., Cz), conclusions about the activity within other areas such as the frontal cortices is only speculative. $\mathrm{Cz}$ is the recommended electrode site for investigating the paired click paradigm, which is why it was selected for the presented research. Future research involving the PCOP paradigm should investigate possible age differences across the frontal electrode sites, as results from these studies could provide evidence that older adults experience differences in sensory gating during attentive stages due to over-recruitment of the frontal cortices.

Second, after removing two participants the total sample size was reduced to 28 , and this slightly impacted the power of the study. Throughout the analysis, one or two participants would not have a distinguishable ERP, and therefore had to be dropped from a given statistical test. This is typical for ERP research, but could be better account for in future research by collecting more participants than suggested based on a power analysis. It is also worth mentioning that future research should collect participants that encompass not only two ends of the age spectrum, but include middle-aged adults. For this study, regression analysis was not selected due to the drastic separation in group age means. Collecting a more representative sample in terms of age variability is recommended, and would add to the limited research investigating age and sensory gating.

Lastly, using time locked events like ERPs only provides a window into the function of the brain. Using continuous measurements, like investigating the presence of 
various brain waves just prior to or immediately after a stimulus is presented, would provide additional information when investigating how age influences both higher order executive functioning and sensory gating.

\subsection{Future Research}

When considering the context of the environment, weaker sensory gating could provide an advantage when older adults are already over-recruiting within their frontal cortices. If information can more easily pass through the attentional filter of older adults, weaker gating may provide a mechanism to ensure attention is directed at unexpected information.

Attentive stage gating differences, like the N100 and P200 gating ratios, should be further investigated. The observation that age-related gating differences were found within the easy but not hard workload suggests that older adults reach a neurological threshold in recruitment during easy workload tasks more quickly than younger adults, but that this difference is somehow mitigated during hard workload. Studies utilizing fMRI or fNIRS technology during sensory gating paradigms would add to this investigation. Further, investigating additional electrode sites along the frontal and parietal cortices would provide further information regarding how attentive processes change with age based on shifts in neural recruitment. 


\section{Appendices}

\section{Appendix A - Demographic and state questions.}

Age:

Gender: $\quad$ Male $\quad$ Female $\quad$ Prefer not to answer

Please circle your responses to the following questions:

Have you previously had a concussion? $\quad$ Yes $\quad$ No

Do you have normal to corrected vision? Yes No

$\begin{array}{lll}\text { Do you have any hearing impairments? } & \text { Yes }\end{array}$

Are right or left-handed? Right $\quad$ Left Both

$\begin{array}{lll}\text { Is English your first language? } & \text { Yes } & \text { No }\end{array}$

1. How alert do you feel right now? (1 - Not at all alert, 10 - Very alert)

$\begin{array}{llllllllll}1 & 2 & 3 & 4 & 5 & 6 & 7 & 8 & 9 & 10\end{array}$

Not at all Neutral Very alert

2. Approximately how many hours of sleep did you get last night?
$0-4$
$5-6$
$7-8$
9 or more hours

3. I last ate...

Within 30 minutes Within the last hour Within 2 hours Over 2 hours ago 
Office of Research Ethics

5110 Human Computer Interaction Bldg | 1125 Colonel By Drive

| Ottawa, Ontario K1S 5B6

613-520-2600 Ext: 4085

ethics@carleton.ca

\section{CERTIFICATION OF INSTITUTIONAL ETHICS CLEARANCE}

The Carleton University Research Ethics Board-B (CUREB-B) has granted ethics clearance for the changes to protocol to research project described below and research may now proceed. CUREB-B is constituted and operates in compliance with the Tri-Council Policy Statement: Ethical Conduct for Research Involving Humans (TCPS2).

Ethics Clearance ID: Project \# 104917 13-094

Principal Investigator: Dr. Chris Herdman

Co-Investigator(s) (If applicable): Dr. Chris Herdman (Primary Investigator)

Dr. Kathleen Van Benthem (Other)

Caidence Paleske (Other)

Project Title: An Evaluation of Workload in Visual Processing and Storage Tasks Using an Auditory Oddball Paradigm: An ERP Study

\section{Funding Source:}

Effective: September 12, 2018

Expires: September 30, 2019.

Upon reasonable request, it is the policy of CUREB, for cleared protocols, to release the name of the PI, the title of the project, and the date of clearance and any renewal(s).

Please email the Research Compliance Coordinators at ethics@carleton.ca if you have any questions.

CLEARED BY:

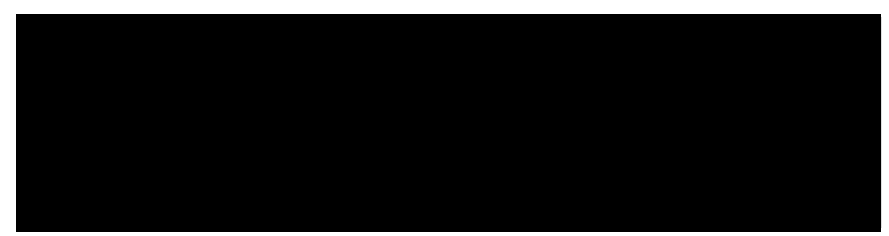

Bernadette Campbell, PhD, Chair, CUREB-B
Date: September 12, 2018 


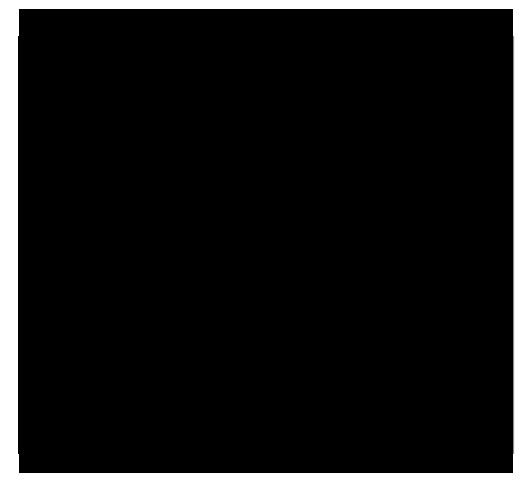

Natasha Artemeva, PhD, Vice Chair, CUREB-B 


\section{References}

Adler, L. E., Pachtman, E., Franks, R. D., Pecevich, M., Waldo, M. C., \& Freedman, R. (1982). Neurophysiological evidence for a defect in neuronal mechanisms involved in sensory gating in schizophrenia. Biological Psychiatry, 17(6), 639654.

Ashan, N. (2016). Creative control: a neurocognitive index of relationships between creativity and attention (Masters dissertation). Carleton University.

Baddeley, A. (2000). The episodic buffer: a new component of working memory?. Trends in Cognitive Sciences, 4(11), 417-423.

Baddeley, A. \& Hitch, G. (1974). Working memory. Psychology of Learning and Motivation, 8, 47-89.

Boutros, N. N., Zouridakis, G., \& Overall, J. (1991). Replication and extension of P50 findings in schizophrenia. Clinical Electroencephalography, 22(1), 40-45.

Boutros, N. N., Reid, M. C., Petrakis, I., Campbell, D., Torello, M., \& Krystal, J. (2000). Similarities in the disturbances in cortical information processing in alcoholism and aging: a pilot evoked potential study. International Psychogeriatrics, 12(4), 513-525.

Callan, D. E., Gateau, T., Durantin, G., Gonthier, N., \& Dehais, F. (2018). Experiment synchrony is related to identification of inattentional deafness in real-world setting. Human Brain Mapping, 39, 2596-2608.

Campbell, J.I. \& Thompson, V.A. (2012). MorePower 9.0 for ANOVA with relational confidence intervals and Bayesian analysis. Behavioural Research Methods, 44(4), 1255-1265.

Causse, M., Chua, Z.K., \& Remy, F. (2019). Influences of age, mental workload, and flight experience on cognitive performance and prefrontal activity in private pilots: a fNIRS study. Scientific Reports, 9, 7688.

Corbetta M, Shulman GL. 2002. Control of goal-directed and stimulus-driven attention in the brain. Nature Reviews Neuroscience, 3, 201-215. 
Crowley, K.E. \& Colrain, I.M. (2004). A review of the evidence for P2 being an independent component process: age; sleep, and modality. Clinical Neurophysiology, 115(4), 732-744.

Daffner, K.R., Chong, H., Sun, X., Tarbi, E.C., Riis, J.L., McGinnis, S.M., \& Holcomb, P.J. (2010). Mechanisms underlying age- and performance-related differences in working memory. Journal of Cognitive Neuroscience, 23 (6), 1298-1214.

Dalecki, A., Croft, \& J. R., Johnstone, S. J. (2011). An evaluation of P50 paired-click methodologies. Psychophysiology, 48(12), 1692-1700.

D'Anselmo, A., Marzoli, D., Brancucci, A. (2016). The influence of memory and attention on the ear advatange in dichotic listening. Hearing Research, 342, 144149.

Davis, S. W., Dennis, N. A., Daselaar, S. M., Fleck, M. S., \& Cabeza, R. (2008). Que PASA? The posterior-anterior shift in aging. Cerebral Cortex, 18(5), 1201-1209.

D’Esposito M., Detre J. A., Alsop D. C., Shin R. K., Atlas S., Grossman M. (1995). The neural basis of the central executive system of working memory. Nature, 378, 279-281.

Dehais, F., Causse, M., Vachon, F., Regis, N., Menant, E., \& Tremblay, S. (2014). Failure to detect critical auditory alerts in the cockpit: evidence for inattentional deafness. Human Factors, 56(4), 631-644.

Delorme, A \& Makeig, S. (2004) EEGLAB: an open source toolbox for analysis of single-trial EEG dynamics (pdf). Journal of Neuroscience Methods, 134, 9-21.

Dempster, F. N. (1992). The rise and fall of the inhibitory mechanism: Toward a unified theory of cognitive development and aging. Developmental Review, 12(1), 4575.

Dien, J. (1998). Issues in the application of the average reference: review, critiques, and recommendations. Behaviour Research Methods, Instruments, and Computers, 30(1), 34-43.

Endsley, M. R. (1995). Toward a theory of situation awareness in dynamic systems. Human Factors, 37(1), 32-64. 
Fjell, A. \& Walhovd, K. (2001). P300 and neurological tests as measures of aging: scalp topography and cognitive changes. Brain Topography, 14(1), 25-40.

Fuest, D.R., Gallinat, J., \& Boutros, N.N. (2007). Range of sensory gating values and testretest reliability in normal subjects. Psychophysiology, 44, 620-626.

Gabard-Durnam, L.J. , Leal, A.S.M., Wilkinson, C.L., \& Levin, A.R. (2018). The Harvard automated processing pipeline for electroencephalography (HAPPE): Standardized processing software for developmental and high-artifact data. Frontiers in Neuroscience: Brain Imaging Methods, https://doi.org/10.3389/fnins.2018.00097.

Gazzaley, A., Cooney, J. W., McEvoy, K., Knight, R. T., \& D’Esposito, M. (2005). Topdown enhancement and suppression of the magnitude and speed of neural activity. Journal of Cognitive Neuroscience, 17(3), 507-517.

Girden, E.R. (1992). ANOVA: repeated measures. Newbury Park, CA: Sage Publications.

Gjini, K., Burroughs, S., Boutros, N.N. (2011). Relevance of attention in auditory sensory gating paradigms in schizophrenia. Journal of Psychophysiology, 22, 60-66.

Gmehlin, D., Kreisel, S., Bachman, S., Weisbrod, M., \& Thomas, C. (2011). Age effects on preattentive and early attentive auditory processing of redundant stimuli: Is sensory gating affect by physiological aging?. The Journals of Gerontology, 66a(10), 1043-1053.

Goodale, M.A. \& Milner, A.D. (1992). Separate visual pathways for perception and action. Trends in Neuroscience, 15(1), 20-25.

Grady, C. L., Maisog, J. M., Horwtiz, B., Ungerleider, L. G., Mentis, M. J., Salerno, J. A., Pietrini, P., Wagner, E., \& Haxby, J. V. (1994). Age-related changes in cortical blood flow activation during visual processing of faces and locations. Journal of Neuroscience, 14(3), 1450-1462.

Guerreiro, M. J. S., Anguera, J. A., Mishra, J., Van Gerven, P. W. M., \& Gazzaley, A. (2014). Age-equivalent top-down modulation during cross-modal selective attention. Journal of Cognitive Neuroscience, 26(12), 2827-2839. 
Guerreiro, M. J. S., \& Van Gerven, P. W. M. (2011). Now you see it, now you don't: Evidence for age-dependent and age-independent cross-modal distraction. Psychology and Aging, 26(2), 415-426.

Hackett, T. (2012). Information flow in the auditory cortical network. Hearing Research, 271, 133-146.

Hall, M.D., Pastore, R.E., Acker, B.E., \& Huang W. (2000). Evidence for auditory feature integration with spatially distributed items. Perception \& Psychophysics, 62(6), 1243-1257.

Hamilton, R.K., Baskin-Sommers, A.R., Newman, J.P. (2014). Relation of frontal N100 to psychopathy-related differences in selective attention. Biological Psychology, 103, 107-116.

Herath P., Klingberg T., Young J., Amunts K., Roland P. (2001). Neural correlates of dual task interference can be dissociated from those of divided attention: an fMRI study. Cerebral Cortex, 11, 796-805

Jerger, K., Biggins, C., \& Fein, G. (1992). P50 suppression is not affected by attentional manipulations. Biological Psychiatry, 31(4), 365-377.

Jovicich, J., Peters, R.J., Koch, C., Braun, J., Chang, L., Ernst, T. (2001). Brain areas specific for attentional load in a motion tracking task. Journal of Cognitive Neuroscience, 13(8), 1048-1058.

Juckel, G., Karch, S., Kawohl, W., Kirsch, V., Jäger, L., Leicht, G., Lutz, J., Stammel, A., Pogarell, O., Ertl, M., Reiser, M., Hegerl, U., Moller, H.J., Mulert, C. (2012). Age effects on the P300 potential and the corresponding fMRI BOLD-signal. NeuroImage, 60(4), 2027-2034.

Kennedy, Q., Taylor, J. L., Reade, G., \& Yesavage, J. A. (2010). Age and expertise effects in aviation decision making and flight control in a flight simulator. Aviation Space and Environmental Medicine, 81(5), 489-497.

Leiva, A., Andrés, P., \& Parmentier, F. B. R. (2015). When aging does not increase distraction: Evidence from pure auditory and visual oddball tasks. Journal of Experimental Psychology:Human Perception and Performance, 41(6), 16121622.

Leiva, A., Parmentier, F.B.R. \& Andrés, P. (2015). Aging increases age-related distraction by auditory oddballs in visual, but not auditory tasks. Psychological Research, 79, 401. 
Li, L., Gratton, C., Fabiani, M., \& Knight, R. T. (2013). Age-related frontoparietal changes during the control of bottom-up and top-down attention: an ERP study. Neurobiology of Aging, 34(2), 477-488.

Lijffijt, M., Lane, S.D., Meier, S.L., Boutros, N.N., Burroughs, S., Steinberg, J.L., Moeller, F.G., Swann, A.C. (2009). P50, N100, and P200 sensory gating: relationships with behavioural inhibition, attention, and working memory. Psychophysiology, 46(5), 1059-1068.

Linden, D.E., Bittner, R.A., Muckli, L., Waltz, J.A., Kriegeskorte, N., Goebel, R., Singer, W., \& Munk, M. (2003). Cortical capacity constraints for visual working memory: dissociation of fMRI load effects in a fronto-parietal network. Neuroimage, 20, 1518-1530.

Lubitz, A.F., Niedeggen, M., Feser, M. (2017). Aging and working memory performance: electrophysiological corrlates of high and low performing eldery. Neuropsychologia, 106, 42-51.

Marois, R. \& Ivanoff, J. (2005). Capacity limits of information processing in the brain. Trends in Cognitive Sciences, 9(6), 296-305.

Mayer, A.R., Hanlon, F.M., Franco, A.R., Teshiba, T.M., Thoma, R.J., Clark, V.P., \& Caive, J.M. (2009). The neural networks underlying auditory sensory gating. Neuroimage, 44(1), 182-189.

McAlister, C. \& Schmitter-Edgecombe, M. (2016). Everyday functioning and cognitive correlate in healthy older adults with subjective cognitive concerns. Clinical Neuropsychology, 30(7), 1087-1103.

McEvoy, L.K., Pellouchoud, E., Smith, M.E., \& Gevins, A. (2001). Neurophysiological signals of working memory in normal aging. Cognitive Brain Research, 11(3), 363-376.

Miller M.W., Rietschel J.C., McDonald C.G., \& Hatfield, B.D. (2011). A novel approach to the physiological measurement of mental workload. International Journal of Psychophysiology, 80, 75-78.

Morrow, D., Leirer, V., Altiteri, P., \& Fitzsimmons, C. (1994). When expertise reduces age differences in performance. Psychology \& Aging, 9(1), 134. 
Moisala, M., Salmela, V., Salo, E., Carlson, S., Vuontela, V., Salonen, O., \& Alho, K. (2015). Brain activity during divided and selective attention to auditory and visual sentence comprehension tasks. Frontiers in Human Neuroscience, 9(86).

O’Connell, R. G., Balsters, J. H., Kilcullen, S. M., Campbell, W., Bokde, A. W., Lai, R., Upton, N., \& Robertson, I. H. (2012). A simultaneous ERP/fMRI of the P300 aging effect. Neurobiology of Aging, 33(10), 2448-2461.

Olincy, A. \& Martin, L. (2005). Diminished suppression of the P50 auditory evoked potential in bipolar disorder subjects with a history of psychosis. American Journal of Psychiatry, 162, 43-49.

Patel, A.D. \& Iversen, J.R. (2007). The linguistic benefits of musical abilities. Trends in Cognitive Sciences, 11(9), 369-372.

Pessoa, L., Gutierrex, E., Bandettini, P.A., Ungerleider, L.G. (2002) Neural correlates of visual working memory: fMRI amplitude predicts task performance. Neuron, 35, 975-987

Rauschecker JP, Tian B. (2000). Mechanisms and streams for processing of "what" and "where" in auditory cortex. Proceedings of the National Academy of Sciences of the United States of America 97:11800-6.

Rensink, R. (2009). Attention: change blindness and inattentional blindness. In W. Banks (Ed.), Encyclopedia of consciousness (Vol. 1, pp. 47-59). New York, NY:Elsevier.

Rodríguez-Aranda, C., \& Sundet, K. (2006). The Frontal Hypothesis of Cognitive Aging: Factor Structure and Age Effects on Four Frontal Tests Among Healthy Individuals. The Journal of Genetic Psychology, 167(3), 269-287.

Simons, D. J., \& Chabris, C. F. (1999). Gorillas in our midst: sustained inattentional blindness for dynamic events. Perception, 28(9), 1059-1074.

Soder, H. \& Potts, G. (2018). Medial frontal cortex response to unexpected motivationally salient outcomes. International Journal of Psychophysiology, 132, 268-276.

Solis-Marcos, I. \& Kircher, K. (2019). Event-related potentials as indices of mental workload while using an in-vehicle information system. Cognition, Technology, and Work, 21(1), 55-67. 
Taylor, J. L., Yesavage, J. A., Morrow, D. G., Dolhert, N., Brooks, J. O., \& Poon, L. W. (1994). The Effects of Information Load and Speech Rate on Younger and Older Aircraft Pilots' Ability to Execute Simulated Air-Traffic Controller Instructions. Journal of Gerontology, 49(5), P191-P200.

Taylor, J. L., O’Hara, R., Mumenthaler, M. S., Rosen, A. C., \& Yesavage, J. A. (2005). Cognitive ability, expertise, and age differences in following air-traffic control instructions. Psychology and Aging, 20(1), 117-133

Taylor, J. L., Kennedy, Q., Noda, A., \& Yesavage, J. A. (2007). Pilot age and expertise predict flight simulator performance - A 3-year longitudinal study. Neurology, 68(9), 648-654.

Thomsen, T., Specht, K., Hammar, A., Nyttingnes, J., Ersland, L., \& Hugdahl, K. (2004). Brain localization of attentional control in different age groups by combing functional and structural MRI. NeuroImage, 22(2), 912-919.

Tsolaki, A., Kosmidou, V., Hadjileontiadis, L., Kompatsiaris, I., \& Tsolaki, M. (2015). Brain source localization of MMN, P300 and N400: Aging and gender differences. Brain Research, 1603, 32-49.

Turner, G. R. \& Spreng, R. N. (2012). Executive functions and neurocognitive aging: dissociable patterns of brain activity. Neurobiology of Aging, 33(4), 826.e1826.e13.

Van Gerven, P. W. M., \& Guerreiro, M. J. S. (2016). Selective Attention and Sensory Modality in Aging: Curses and Blessings. Frontiers in Human Neuroscience, 10, 147.

Van Benthem, K., Cebulski, S., Herdman, C.M., Keillor, J. (2018). An EEG braincomputer interface approach for clsasifying vigilance states in humans: a gamma band focus suppors low misclassification rates. International Journal of HumanComputer Interaction, 24(3), 226-237.

Weber-Fox, C., Leonard, L.B., Wray, A.H., Tomblin, J.B. (2010). Electrophysiological correlates of rapid auditory and linguistic processing in adolescents with specific language impairment. Brain and Language, 115(3), 162-181. 
Winkler, I., Brandl, S., Horn, F., Waldburger, E., Allefeld, C., \& Tangermann, M (2014). Robust artifactual independent component classification for BCI practitioners. Journal of Neural Engineering, 11, doi:10.1088/1741-2560/11/3/035013.

Yesavage, J. A., Jo, B., Adamson, M. M., Kennedy, Q., Noda, A., Hernandez, B., Taylor, J. L. (2011). Initial Cognitive Performance Predicts Longitudinal Aviator Performance. Journals of Gerontology Series B: Psychological Sciences and Social Sciences, 66B(4), 444-453.

Yesavage, J. A., Taylor, J. L., Mumenthaler, M. S., Noda, A., \& O'Hara, R. (1999). Relationship of age and simulated flight performance. Journal of the American Geriatrics Society, 47(7), 819-823.

Yoo S. S., Paralkar G., Panych L. P. (2004). Neural substrates associated with the concurrent performance of dual working memory tasks. International Journal of Neuroscience, 114, 613-631.

Yurgil, K.A. \& Golob, E.J. (2013). Cortical potential in an auditory oddball task reflect individual differences in working memory capacity. Psychophysiology, 50(12), 1263-1274. 